\title{
LES STRONGYLIDÉS DU RHINOCÉROS AFRICAIN (RHINOCEROS BICORNIS)
}

\author{
Par M. NEVEU-LEMAIRE
}

Les parasites du rhinocéros africain, Rhinoceros bicornis, sont encore fort peu connus et, jusqu'ici, une seule espèce de Strongylidx: Kiluluma stylosa (von Linstow, 1907), a été signalée chez ce grand mammifère (1). Les rhinocéros ne sont point, en effet, des animaux de capture courante, comme les éléphants que l'on chasse pour l'ivoire de leurs défenses ; s’ils sont tués par des indigènes ou par un sportsman, n'ayant d'autre but que d'ajouter un gibier rare à son tableau de chasse, ils ne sont, pas plus dans un cas que dans l'autre, l'objet de recherches parasitologiques et c'est ce qui explique pourquoi leur faune parasitaire a été si peu étudiée.

Aussi, suis-je particulièrement reconnaissant à mon ami, le professeur E. Brumpt, d'avoir bien voulu me communiquer de nombreux échantillons de nématodes, recueillis par lui en 1902, dans la région du lac Rodolphe, à l'autopsie d'un rhinocéros, tué par le vicomte du Bourg de Bozas, dont il était le collaborateur scientifique, au cours de sa mission en Afrique.

Je tiens aussi à adresser mes remerciements au professeur Henry d'Alfort, qui a bien voulu me communiquer les riches documents de son laboratoire et dont les conseils m'ont été précieux.

Tous les spécimens que j'ai examinés appartiennent à la famille des Strongylidx; ce sont, pour la plupart, des genres nouveaux et, tous, des epèces nouvelles; ils peuvent être répartis dans les trois sous-familles des Strongylinæ, des Bunostominæ et des Cylicostominæ, ainsi que dans une nouvelle sous-famille que j'appellerai les Amirinx.

\section{Strongylinæ}

Les Strongylinæ sont caractérisés par une bourse caudale à côtes ventrales soudées, à côtes latérales nettement séparées, à côtes dor-

(1) H. A. Baylis a décrit en 1919, sous le nom de Crossocephalus longicaudalus, un nématode appartenant à la famille des Atractida et parasite d'une autre espèce de rhinocéros, Rhinoceros sumatrensis, de la Péninsule Malaise.

Annales de Parasitologie, T. II, N² 2 - Avril 1924, p. 121. 
sales externes naissant d'un tronc commun avec la dorsale médiane, chaque branche de cette dernière étant tridigitée. La vulve est située au voisinage du tiers postérieur du corps et les utérus sont opposés.

Nous avons rencontré deux espèces parasites du rhinocéros africain, appartenant à cette sous-famille; nous classons l'une d'elles dans le genre Quilonia C. Lane, 1914; l'autre nous paraît être le type d'un genre nouveau.

\section{Genre Quilonia C. Lane, 1914}

Synonymie. - Evansia Railliet et Henry, 1913, non Evansia Scott, 1906 (Copépode) ; Nematevansia Ihle, 1919.

Le genre Quilonia a été créé par Clayton Lane, en 1914, pour un strongylidé trouvé dans le cæcum de l'éléphant d'Asie, Elephas maximus, à Travancore. Il a donné à ce nématode le nom de $Q$. quilona. Or Railliet et Henry avaient décrit, l'année précédente, en 1913, cette même espèce, déjà figurée en 1910 par Evans et Rennie, sous le nom d'Evansia Renniei. L'espèce de C. Lane tombe donc de ce fait en synonymie, mais son nom générique doit subsister, le nom d'Evansia étant préoccupé et ayant été donné en 1906 par Scott à un crustacé copépode.

D'après Railliet, la constitution de la capsule buccale de ce genre le rapproche des Cylicostominx, mais la conformation de la bourse caudale, caractère qu'il juge avec raison beaucoup plus important, comme élément de classification, que la structure de l'appareil digestif, le fait rentrer parmi les Strongylinx.

Diagnose. - Strongylinæ à extrémité antérieure droite. La bouche, terminale, est entourée de deux papilles latérales sessiles et de quatre papilles submédianes proéminentes. La coronule externe est formée de lamelles peu nombreuses, divergentes et incurvées à leur sommet. En coupe optique, on voit la cuticule qui les forme se continuer postérieurement pour rejoindre le contour cuticulaire de l'œsophage, sans rejoindre la capsule buccale. La capsule buccale, rappelant celle des Cylicostominæx, est très courte, forme plus ou moins un anneau circulaire et est largement séparée de la cavité buceale par du parenchyme. L'œesophage est à peu près cylindrique. Les valvules intestinales sont normales.

Mále. - Le lobe dorsal de la bourse caudale est plus développé que les autres. La côte dorsale est divisée en deux branches, dont chacune est tridigitée. Les spicules sont égaux et semblables, ayant chacun une pointe falciforme. La pièce accessoire est plus atté- 
nuée en avant qu'en arrière et forme une gouttière à concavité ventrale; ses ailes latérales sont triangulaires.

Femelle. - La vulve est située vers le quart postérieur du corps. Les utérus sont opposés, divergents, à ovéjecteurs bien développés. En réalité les ovéjecteurs sont seuls opposés, car l'utérus postérieur se replie aussitôt pour se diriger en avant et cheminer antérieurement à côté de l'utérus antérieur.

TyPe du Genre. - Quilonia Renniei (Railliet et Henry, 1913), du cæcum de l'éléphant d'Asie, Elephas maximus.

\section{Quilonia rhinocerotis n. sp.}

(Planche IX)

Description. - Le corps est cylindroïde, de couleur blanchàtre et la cuticule est striée transversalement. L'extrémité antérieure étant en mauvais état chez tous les exemplaires examinés, il est difficile de distinguer nettement ses caractères. La bouche, terminale, est entourée d'une coronule externe, formée de quelques lames seulement, longues, déchiquetěes, proéminentes et recourbées à la périphérie. La capsule buccale est excessivement courte, ayant la forme d'un petit anneau circulaire. L'œsophage, de longueur moyenne, est à peu près cylindrique, légèrement renflé à sa partie postérieure et entouré par l'anneau nerveux situé un peu en avant de son milieu ; son extrémité postérieure est munie de valvules. Le pore excréteur est situé un peu en arrière de la naissance de l'intestin, qui est formé de cellules bien marquées.

Mâle. - Le mâle présente presque le même diamètre dans toute son étendue, l'extrémité antérieure étant un peu plus étroite. Les deux extrémités paraissent tronquées et, même à l'œil nu, on aperescit la bourse caudale, cupuliforme. Le lobe dorsal de celle-ci est très développé. Les côtes dorsales externes naissent d'un volumineux trone commun aqec la côte dorsale médiane. Cette dernière, épaisse à sa naissance et plus longue que toutes les autres, n'est dédoublée qu'à peu de distance de l'endroit où chacune de ses branches se divise d'abord en deux ramifications, la plus interne se subdivisant à son tour en deux rameaux, dont l'interne est le plus grand. Les côtes ventrales sont soudées et peu développées. Des trois côtes latérales, la latérale externe est la plus petite et la latérale postérieure la plus grande. Les spicules sont égaux, légèrement recourbés, finement striés et à extrémité falciforme. La pièce accessoire est incurvée ventralement et présente la disposition typique du genre. 
Femelle. - La femelle, plus grande que le mâle, présente son plus grand diamètre vers le milieu du corps, elle est atténuée à son extrémité antérieure et pointue à son extrémité postérieure. L'anus est situé à la naissance de cette pointe très fine. La vulve, assez éloignée de l'anus, se trouve vers le quart postérieur du corps ; elle est petite et circulaire. Au vagin, à peine distinct, aboutissent les ovéjecteurs, qui viennent l'un de la partie antérieure, l'autre de la partie postérieure. L'utérus postérieur, au niveau du point où il aboutit à l'ovéjecteur, remonte pour cheminer parällèlement à l'utérus antérieur et les deux utérus se dirigent vers l'extrémité céphalique. Les ovaires qui leur font suite décrivent des circonvolutions jusqu'un peu au delà du tiers antérieur du corps.

Les œufs, qui se trouvent dans les ovéjecteurs et dans les utérus, sont ovoïdes, à cuticule mince et déjà segmentés.

\section{Dimensions DE QUILONIA RHINOCEROTIS}

Largeur de la tète

Ecartement des stries cuticulaires au niveau de la tête . $40 \mu$

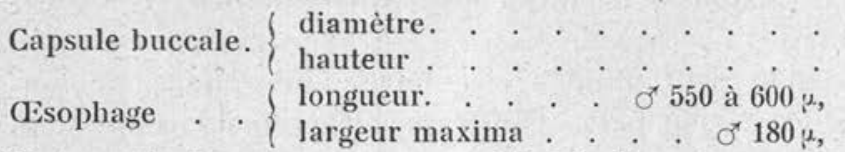

Distance de l'anneau nerveux au début de l'œsophage. . . 180 à 200 :

Distance de l'extrémité antérieure au pore excréteur. . . $880 \mu$

ơ $125 \mu$, ㅇ $150 \mu$

ơ $20 \mu$, , 우․ $25 \mu$

○ 610 à $700 \mu$

○ 200 à 225

\section{Mâle}

Longueur .

Largeur maxima .

Longueur de la côte dorsale .

Longueur des spicules

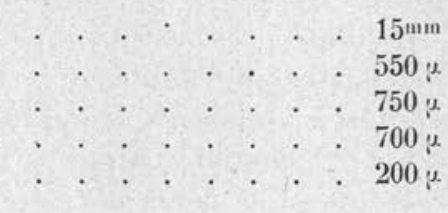

Femelle

Longueur .

$23^{\mathrm{mm}}$

Largeur maxima .

$800 \mu$

Distance de l'anus à l'extrémité postėrieure . . . . . $2^{\mathrm{mm}, 6}$

Distance de la vulve à l'extrémité postérieure . . . . $5^{\mathrm{mm}}, 7$

Dimensions des œufs dans l'utérus .

$70 \mu \times 45 \mu$

Hôte. - Cette espèce est très commune dans le gros intestin du rhinocéros d'Afrique, Rhinoceros bicornis L.

Localité. - Voisinage de la rivière Ousnée, affluent du fleuve Omo, dans la région du lac Rodolphe (Afrique orientale). 


\section{Genre Paraquilonia n. $\mathrm{g}$.}

Diagnose. - Strongylinæ à extrémité antérieure droite. La bouche, terminale, est entourée de six lèvres épaisses et arrondies: deux dorsales et deux ventrales portant chacune une papille proéminente avec un bouton terminal et deux latérales, une latérale droite et une latérale gauche dépourvues de papille. La coronule est rudimentaire. La capsule buccale est très courte et forme un anneau à peu près circulaire. L'œsophage est presque cylindrique. Les valvules intestinales sont normales.

Mále. - La bourse caudale affecte la même disposition que dans le genre Quilonia : développement assez grand du lobe dorsal; côte dorsale divisée en deux branches dont chacune est tridigitée. Les spicules, grêles, sont égaux et semblables. Il existe une pièce accessoire.

Femelle. - La vulve est située vers le quart postérieur du corps. L'appareil génital est disposé comme chez les Quilonia : les utérus sont opposés et divergents, avec des ovéjecteurs bien développés ; ceux-ci étant à vrai dire seuls opposés, car l'utérus postérieur se replie de suite pour se diriger antérieurement.

Type de GEnRe. - Paraquilonia Brumpti n. g., n. sp., du gros intestin du rhinocéros africain, Rhinoceros bicornis.

\section{Paraquilonia Brumpti n. g., n. sp.}

(Planche X)

Description. - Le corps est cylindroïde, de couleur blanchâtre, revêtu d'une cuticule nettement striée transversalement sur toute son étendue. La tête présente la disposition que nous venons d'indiquer : bouche entourée de six lèvres, dont quatre, deux dorsales et deux ventrales portent des papilles saillantes et dont deux, latérales, n'en portent point. La coronule est à peine distincte. La capsule buccale est très réduite et de forme annulaire. Les glandes céphaliques sont très développées. L'œsophage est de longueur moyenne et presque cylindrique, à peine rétréci au niveau de l'anneau nerveux qui l'encercle un peu en arrière de son tiers antérieur; il présente des valvules à son extrémité postérieure. Le pore excréteur est très voisin de cette extrémité. Les cellules de l'intestin sont bien distinctes.

Mâle. - Le mâle présente sensiblement le même diamètre sur toute son étendue et ses extrémités semblent tronquées. La bourse caudale est assez développée pour qu'on puisse la distinguer à l'œil 
nu ; elle est cupuliforme, avec un lobe dorsal allongé. La côte dorsale, relativement épaisse à sa base, donne naissance aux dorsales externes, puis se divise en deux branches, dont chacune est tridigitée. Des trois digitations, l'externe est la plus longue, puis l'interne, la médiane étant la plus courte. Des trois côtes latérales, la latérale externe est la plus petite; les deux autres sont sensiblemen égales. Les spicules sont égaux et grêles et il existe une pièce accessoire.

Femelle. - La femelle est un peu plus grande que le mâle, avec son plus grand diamètre dans la région moyenne du corps. L'extrémité antérieure est à peine atténuée et tronquée, tandis que l'extrémité postérieure se termine par une pointe effilée. L'anus est situé à la naissance de cette pointe. La vulve se présente sous l'aspect d'un petit orifice circulaire, placé vers le quart postérieur du corps. Dans un vagin très court viennent déboucher deux ovéjecteurs, venant l'un de la partie antérieure, l'autre de la partie postérieure. L'utérus postérieur, au niveau du point où il aboutit à l'ovéjecteur, remonte pour cheminer parallèlement à l'utérus antérieur et tous deux se dirigent vers l'extrémité céphalique. Les ovaires qui leur font suite décrivent de nombreuses circonvolutions jusque vers le quart antérieur du corps.

Les œufs, renfermés dans les ovéjecteurs ou les utérus, sont ovoïdes, avec une cuticule mince et ont subi un commencement de segmentation.

\section{DIMENSIONS DE PARAQUILONIA BRUMPTI}

Largeur de la tête

Hauteur des lèvres sans les papilles.

Ecartement des $\}$ au niveau de la tête . . . . . . 40

stries cuticulaires \{ dans la région moyênne du corps . $30 \mu$

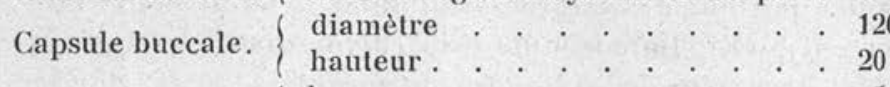

Esophage. $\quad\left\{\begin{array}{l}\text { longueur. } \\ \text { largeur maxima }\end{array}\right.$ Distance de l'anneau nerveux au début de l'œsophage.

Distance du pore excréteur à l'extrémité antérieure
175 à $200 \mu$

$40 \mu$

120 à $150 \mu$

$20 \mu$

$\sigma^{7} 600 \mu$, $ᄋ 650 \mu$ $200 \mu$

o $: 00 \mu$, \& $250 \mu$

$810 \mu$

Màle

Longueur

$14 \mathrm{~mm}$

Largeur maxima . . . . . . . . . . . . . $525 \%$

Longueur de la côte dorsale . . . . . . . . . . $500 \mu$

Longueur des spicules . . . . . . . . . . . . $800 \mu$

Longueur du gorgeret . . . . . . . . . . $100 \%$ 
Femelle

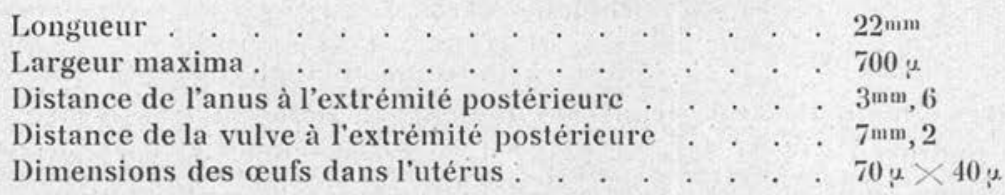

Hôte. - Cette espèce a été trouvée en petit nombre dans le gros intestin du rhinocéros d'Afrique, Rhinoceros bicornis L.

Localité. - Voisinage de la rivière Ousnée, affluent du fleuve Omo, dans la région du lac Rodolphe (Afrique orientale).

\section{Bunostominæ}

Les Bunostominæ sont caractérisés par une bourse caudale à côtes ventrales soudées, à côtes latérale médiane et latérale postérieure soudées sur une longueur plus ou moins grande, leur partie libre n'atteignant pas l'intersection de la côte latérale externe, plus ou moins séparée des deux autres; les côtes dorsales externes naissent d'un tronc commun, parfois très court, avec la dorsale médiane, chaque branche de cette dernière étant bidigitée ou tridigitée. La vulve est comprise entre la moitié et le tiers antérieur du corps et les utérus sont opposés,

Nous avons rencontré, parmi les parasites du rhinocéros africain, une espèce nouvelle appartenant au genre Grammocephalus.

\section{Grammocephalus Railliet et Henry, 1910}

Synonymie. - Strongylus Goeze, 1782 , pro parte; Sclerostoma Rudolphi, 1809, pro parte.

Diagnose. - Bunostominæ à extrémité céphalique relevée vers la face dorsale. La capsule buccale offre à son entrée deux lames ventrales et porte à son fond des lancettes très aiguës. Le mâle a des spicules courts et épais. La femelle est plus petite que le mâle, ce qui est exceptionnel parmi les nématodes; la vulve est située vers le milieu du corps.

TYPE DU GENRE. - Grammocephalus clathratus (Baird, 1868), des canaux biliaires de l'éléphant d'Afrique, Loxodonta africana. 


\section{Grammocephalus intermedius n. sp.}

(Planche XI)

Description. - Le corps est cylindrö̈de, blanchâtre et à cuticule très finement striée transversalement. La tête est très légèrement inclinée vers la face dorsale. La capsule buccale présente deux lames sur son bord ventral et au fond des lancettes bidenticulées. L'œsophage est long et' grêle ; de la naissance de l'intestin part un diverticule qui remonte jusque vers le tiers antérieur de l'œsophage, un peu en arrière de l'anneau nerveux.

Mâle. - Le mâle, plus grand que la femelle, est atténué en avant; les lobes latéraux de la bourse caudale sont très développés. Une papille prébursale conique se trouve en avant des côtes ventrales qui sont isoudées. Les côtes latérales sont volumineuses ; la latérale externe se détache nettement des deux autres qui sont soudées à leur base sur environ moitié de leur longueur. Le lobe dorsal étant très endommagé, je n'ai pu voir l'extrémité des côtes dorsales. Les spicules isont courts et épais.

Femelle. - La femelle, atténuée en avant, a un diamètre moindre que celui du mâle, mais, n'ayant pas eu à ma disposition d'exemplaire entier, je n'ai pu voir l'extrémité postérieure, pas plus que la situation de la vulve.

Bien que je n'aie pu examiner que des spécimens en mauvais état, cette espèce me paraît différer des deux espèces décrites jusqu'ici et provenant des canaux biliaires des éléphants d'Asie et d'Afrique, par ses dimensions, par la forme des lancettes de la capsule buccale, par la conformation des spicules, enfin par son habitat.

\section{DIMENSIONS DE GRAMMOCEPHALUS INTERMEDIUS}

Ecartement des stries cuticulaires . . . . . . . . . . 10 Hauteur de la capsule buccale. . . . . . . . $500 \mu$

Longueur de l'osophage. . . . . . . . . . . . $3^{\mathrm{mm}, 5}$

Distance de l'extrémité de la tète à l'anneau nerreux . $\quad 1 \mathrm{~mm}, 2$

Longueur du diverticule intestinal . . . . . . . $2^{\mathrm{mm}}, 55$

Mâle

Longueu

$52^{\mathrm{mm}}$

Largeur maxima $1 \mathrm{~mm}, 6$

Longeur des spicules. $1 \mathrm{~mm}, 35$

Femelle

Largeur maxima 
Hôte. - Ce strongle est le plus grand de ceux qui composaient le lot que j'ai examiné; il se trouvait en petite quantité dans le gros intestin du rhinocéros d'Afrique, Rhinoceros bicornis L.

Localité. - Voisinage de la rivière Ousnée, affluent du fleuve Omo, dans la région du lac Rodolphe (Afrique orientale).

\section{Amirinæ n. s.-f.}

Dans la classification des Strongylidx, établie par A. Railliet et A. Henry et à laquelle nous nous conformons dans ce travail, il était déjà difficile de placer le genre Amira C. Lane, 1914; or la découverte de deux espèces voisines, l'une déerite par Khalil, en 1922, sous le nom d'Amira sameera, et l'autre que nous avons rencontrée chez le rhinocénos africain et que nous allons décrire, vient encore compliquer la question et il devient impossible de faire rentrer ces strongles dans les subdivisions établies jusqu'ici. La conformation de la bourse caudale du mâle, chez lequel les côtes latérales sont soudées, rapproche ces nématodes des Bunostomi$n x$, tandis que la situation de la vulve proche de l'anus et le parallélisme des utérus chez la femelle les placenaient parmi les Cylicostominæ. Ces considérations nous ont amené à créer une sous-famille nouvelle, les Amirinæ.

Les Amirinæ sont donc caractérisés par une bourse caudale à côtes ventrales grêles et soudées, précédées d'une papille prébursale ayant la forme d'une côte longue ét mince, à côtes latérales presque complètement soudées et formant une masse compacte, à côte dorsale externe naissant d'un tronc commun avec la dorsale médiane, chaque branche de cette dernière étant tridigitée. La vulve est très rapprochée de l'anus; le vagin est très long et les utérus sont parallèles.

Cette sous-famille comprend des strongles chez lesquels l'extrémité antérieure est très caractéristique, avec un œsophage en forme de sablier, mais dont la bourse caudale est bien différente suivant les espèces. Aussi croyons-nous devoir répartir les trois espèces connues jusqu'ici en deux genres :

$1^{\circ}$ Le genre Amira Lane, 1914, dans lequel l'extrémité antérieure est droite et le lobe dorsal de la bourse caudale considérablement allongé. L'espèce-type est Amira pileata (Railliet, Henry et Bauche, 1914) de l'éléphant d'Asie.

$2^{\circ}$ Le genre Khalilia n. g., que nous dédions à M. M. Khalil et dans lequel l'extrémité antérieure est légèrement déjetée dorsale- 
ment, tandis que le lobe dorsal de la bourse caudale est moins développé que les lobes latéraux. L'espèce-type est Khalilia rhinocerotis n. g., n. sp., du rhinocéros africain et l'autre espèce est Khalilia sameera (Khalil, 1922) de l'éléphant d'Afrique.

\section{Genre Khalilia n. g.}

DIAGNose. - Amirinæ à extrémité antérieure légèrement inclinée vers la face dorsale. La bouche, également déjetée dorsalement, est entourée d'une coronule externe à lames convergentes et possède une coronule interne sur le bord antérieur de la capsule buccale, qui est courte, subglobuleuse et plus profonde ventralement que dorsalement. L'œsophage est très court et en forme de sablier, l'anneau nerveux entourant sa partie resserrée; il présente des valvules à son extrémité postérieure.

Mâle. - La bourse caudale du mâle est conformée sur le type de celle des Bunostominæ. Les lobes latéraux sont très développés et soutenus par les côtes ventrales relativement petites, en avant desquelles se trouve une papille prébursale longue et mince ressemblant à une côte, et par les côtes latérales, formant de chaque côté un trone volumineux. Le lobe dorsal, plus petit que les lobes latéraux, est maintenu par une côte dorsale au tronc épais, donnant naissance aux côtes dorsales externes et se divisant aussitôt en deux branches, dont chacune est tridigitée. Les spicules sont long's, grêles et sinueux; il existe une pièce accessoire.

Femelle. - La vulve est rapprochée de l'anus. Le vagin est très long; les ovéjecteurs et les utérus sont parallèles.

TYPE DU GENRE. - Khalilia rhinocerotis n. g., n: 'sp., du gros intestin du rhinocéros africain. Rhinoceros bicornis.

Khalilia rhinocerotis n. g., n. sp.

(Planche XII)

Description. - Le corps est cylindroïde, à peine atténué en avant et de couleur blanchâtre. La cuticule est très finement striée transversalement au niveau de l'extrémité antérieure; à la hauteur de l'anneau nerveux apparait en outre une striation transversale plus grossière, qui se continue sur le reste du corps et se superpose à la précédente, subsistant plus ou moins nettement dans l'intervalle des larges stries.

L'extrémité antérieure est très légèrement inclinée dorsalement. La bouche est limitée par une coronule externe formée d'une qua- 
rantaine de lamelles convergentes, terminées par une petite dent. La capsule buccale est courte, subglobuleuse, plus profonde ventralement que dorsalement, bordée antérieurement par une coronule interne constituée par de petites denticulations, dont le nombre parait égal à celui des lamelles de la coronule externe.

L'œesophage est très court et en forme de sablier; les deux renflements qu'il présente sont séparés par l'anneau nerveux qui l'encercle au niveau de sa partie rétrécie. Le renflement postérieur est plus long et un peu plus large que l'àntérieur, dont la paroi externe est épaissie et striée transversalement, disposition qui permet de distinguer cette espèce de celle décrite par Khalil. La face interne de l'œsophage est fortement chitinisée; sa lumière se dilate en forme d'entonnoir dans sa portion antérieure, de façon à communiquer largement avec la capsule buccale. La partie postérieure de l'œsophage est munie de valvules.

Mâle. - Les lobes latéraux de la bourse caudale sont plus développés que le lobe dorsal. Les côtes ventrales sont soudées et relativement petites; en avant de celles-ci se trouve une longue et mince papille prébursale. Par contre les côtes latérales forment de chaque côté un trone volumineux, dont les trois divisions sont ainsi disposées : la côte latérale externe, moins grosse et moins longue que les autres, en eist séparée sur environ moitié de sa longueur; les côtes latérales médiane et postérieure, très développées, sont soudées sur toute leur longueur sauf tout à fait à leur extrémité. Les deux trones latéraux forment done, en se rapprochant, comme dans le genre Bunostomum, une sorte de pince à mors latéraux. Le tronc de la côte dorsale médiane est moins épais que celui des côtes latérales ; les côtes dorsales externes, petites, s'en détachent au niveau de la bifurcation de ce tronc en deux branches, qui présentent chacune trois digitations peu développées; la plus longue est la digitation interne, la plus courte, la médiane. Les bords postérieurs des lobes latéraux de la bourse caudale sont finement striés. Le cône génital est saillant. Les spicules, longs et grêles, sont recourbés et sinueux, de sorte qu'il est impossible de les mesurer exactement ; la pièce accessoire est assez réduite.

Femelle. - La femelle est légèrement atténuée à l'extrémité postérieure, terminée par une petite pointe en avant de laquelle se trouve l'anus. La vulve en est très rapprochée. Le vagin, très long, se dirige antérieurement, ainsi que les ovéjecteurs et les utérus qui sont parallèles. Les ovaires se replient vers le tiers antérieur du corps. Les œufs sont segmentés. 


\section{DIMENSIONS DE KHALILIA RHINOCEROTIS}

Largeur de la tête

Hauteur de la coronule externe . . . . . . . . . . . 80

$300 \mu$

Ecartement des ( au niveau de l'œsophage . . . . . . . $30 \mu$

stries cuticulaires $\{$ vers la région moyenne du corps . . . . $60 \mu$

Capsule buccale. $\left\{\begin{array}{l}\text { diamètre dans la région moyenne . . . . } \quad 200 \% \\ \text { lauteur du còté dorsal. }\end{array}\right.$

hauteur du côté ventral. . . . . . . . $75 \mu$

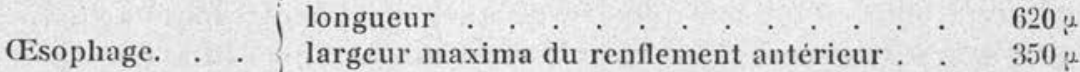

largeur maxima du renflement postérieur. . $400 \mu$

Distance de l'anneau nerveux au début de l'œsophage. . . . . $180 \%$

Maile

Longueur

$11 \mathrm{~mm}$

Largeur maxima .

$730 \mu$

$800 \mu$

Longueur de la còte dorsale

Longueur des côtes latérales.

Longueur du gorgeret

Femelle

Longueur . . . . . . . . . . . . . . . . . $18^{\mathrm{mm}}$

Largeur maxima . . . . . . . . . . . . . . $950 \mu$

Distance de l'anus à l'extrémité poslérieure . . . . . . 600 แ.

Distance de la vulve à l'extrémité postérieure . . . . . $860 \%$

Longueur du vagin . . . . . . . . . . . . . . $3^{\mathrm{mm}, 6}$

Dimensions des œufs dans l'utérus. . . . . . . . . $50 \mu \times 30 \mu$

Hôte. - Les deux exemplaires, mâle et femelle, que nous avons rencontrés proviennent du gros intestin du rhinocéros d'Afrique, Rhinoceros bicornis $\mathrm{L}$.

Localité. - Voisinage de la rivière Ousnée, affluent du fleuve Omo, dans la région du lac Rodolphe (Afrique orientale).

\section{Cylicostominæ}

Les Cylicostominæ sont caractérisés par une bourse caudale à côtes ventrales soudées, à côtes latérales séparées, à côtes dorsales externes naissant isolément et à côte dorsale médiane divisée, chaque branche étant généralement tridigitée. Toutefois les deux rameaux externes de chaque branche peuvent être soudés jusqu'à leur pointe, à peine bifurquée, en sorte que chaque branche 
de la côté dorsale parait seulement bidigitée. La vulve est située près de l'anus et les utérus sont parallèles.

Les nématodes, trouvés dans le gros intestin du rhinocéros africain, comprennent de nombreuses espèces appartenant à cette sous-famille. Contrairement aux Cylicostominæ des équidés, ou aux représentants du genre Murshidia Lane, 1914, avec lesquels elles ont de nombreuses affinités, en particulier par la disposition de la bouche, les espèces que j'ai observées chez le rhinocéros ont une bourse caudale, dont les branches de la côte dorsale sont bidigitées, les rameaux externes étant confondus en un seul, sauf tout à fait à leur extrémité, disposition qui les rapproche de certaines espèces parasites de l'éléphant d'Afrique. Etant donné le nombre considérable d'espèces appartenant à cette sous-famille, je crois qu'il y aurait avantage, en se basant sur les caractères que nous venons d'indiquer, à la subdiviser en deux tribus :

$1^{\circ}$ Les Cylicostomer, chez lesquels chaque branche de la côte dorsale médiane est tridigitée, comme dans les genres Cylicostomum, Murshidia, etc.

$2^{\circ}$ Les Pteridopharyngex, chez lesquels chaque branche de la dorsale médiane est seulement bidigitée, comme dans le genre Pteridopharynx, créé par Clayton Lane en 1921, le genre Memphisia établi, par M. Khalil en 1922 et deux nouveaux genres dont nous allons donner la diagnose.

La tribu des Pteridopharyngex $n$. t. forme ainsi un groupe homogène ne renfermant jusqu'ici que des parasites de l'éléphant d'Afrique et du rhinocéros africain. Toutes les espèces de Cylicostominx, que nous avons observées chez ce dernier animal, rentrent dans ce groupe et sont nouvelles ; l'une d'elles appartient au genre Pteridopharynx, une autre au genre Memphisia et les autres sont réparties dans deux genres nouveaux.

C'est viaisemblablement à cette tribu que l'on doit rattacher le genre Kiluluma, créé par Skrjabin en 1916, pour le seul strongle du rhinocéros africain jusqu'ici connu K. stylosa, décrit en 1907 par von Linstow sous le nom de Deletrocephalus stylosus.

\section{Genre Pteridopharynx C. Lane, 1921, M. Khalil emend.}

Ce genre, dit C. Lane, se rapproche du genre Murshidia (1) Lane, 1914 et n'en est séparé que par la structure de la cuticule œsopha-

(1) C. Lane place les genres Pleridopharynx et Murshidia parmi les Esophagostomine: nous les considérons, avec A. Railliet et A. Henry, comme appartenant à la sous-famille des Cylicostomine. 
gienne, la fusion presque complète des deux branches externes de la côte dorsale, la délicatesse des spicules et la proximité de la vulve et de l'anus.

Diagnose. - Cylicostominæ à extrémité antérieure droite. La bouche, terminale, est entourée d'une coronule externe, dont les lames sont de longueur variable. La cavité buccale a la forme d'un entonnoir renversé, sa cuticule venant en contact avec l'anneau presque circulaire qui forme la capsule buccale. L'œesophage est court et encerclé, au niveau de son tiers supérieur, par l'anneau nerveux. La structure plumeu'se de la cuticule œesophagienne est considérée par Khalil comme un caractère spécifique et peut exister ou non.

Mâle. - La côte latérale postérieure porte une proéminence sur son bord postérieur. Des trois rameaux de chaque branche de la côte dorsale de la bourse caudale, les deux externes sont complètement fusionnés, sauf tout à fait à leur extrémité. Les spicules sont égaux et semblables et il existe une pièce accessoire.

Femelle. - La vulve est très rapprochée de l'anus. Le vagin se dirige antérieurement ainsi que les deux branches utérines qui sont parallèles, chacune étant pourvue d'un ovéjecteur.

TYPE DU GENRE. - Pteridopharynx africana C. Lane, 1921, de l'estomac de l'éléphant d'Afrique, Loxodonta africana.

\section{Pteridopharynx omoensis n. sp.}

\section{(Planche XII)}

Description. - Le corps, cylindroïde, à peu près de même diamètre dans toute sa longueur, est de couleur blanchâtre. La cuticule est assez finement striée transversalement. La bouche, terminale, est entourée de quatre papilles, une dorsale, une ventrale, une latérale droite et une latérale gauche, surmontées chacune d'un petit prolongement en forme de bouton. La coronule externe est formée de lames convergentes, de longueur variable, partant de la paroi interne de la capsule buccale et se rejoignant par leur extrémité libre. Il existe en outre une coronule interne limitant le bord antérieur de la capsule buccale, très courte et annulaire. Les glandes cervicales sont bien développées. L'œsophage, très court, est globuleux; il est resserré au niveau de l'anneau nerveux qui l'entoure un peu au-dessous de son tiers antérieur ; son plus grand diamètre est situé au niveau de son tiers postérieur. Il y a des valvules à la naissance de l'intestin, dont les cellules sont assez distinctes. 
Màle. - Aucun exemplaire mâle n'a été observé.

Femelle. - L'extrémité antérieure de la femelle est tronquée, tandis que son extrémité postérieure se termine par une pointe effilée. L'anus est situé à la naissance de cette pointe et, un peu en avant, s'ouvre l'orifice vulvaire, en un point où la cuticule forme une saillie. Dans le vagin débouchent deux ovéjecteurs parallèles, auxquels aboutissent les utérus, également parallèles. Les ovaires ne décrivent point de circonvolutions, mais forment seulement des anses, qui ne dépassent pas le tiers antérieur du corps.

Les ovéjecteurs et les utérus des exemplaires examinés ne contenaient point d'œufs complètement développés.

\section{DIMENSIONS DE PTERIDOPHARYNX OMOENSIS}

Largeur de la tête.

Capsule buccale. $\left\{\begin{array}{l}\text { diamètre } \\ \text { hauteur. }\end{array}\right.$

CEsophage.

Distance de l'anneau nerveux au début de l'œsophage.

Femelle

Longueur .

Largeur maxima .

Distance de l'anus à l'extrémité postérieure .

Distance de la vulve à l'extrémité postérieure
$250 \mu$ à 275 «

$115 u$

$40 \mu$

450 à $480 \mu$

230 à 250

$150 \mu$

Hôte. - Les deux exemplaires femelles que j'ai examinés proviennent du gros intestin du rhinocéros d'Afrique, Rhinoceros bicornis $\mathrm{L}$.

Localité. - Voisinage de la rivière Ousnée, affluent du fleuve Omo, dans la région du lac Rodolphe (Afrique orientale).

\section{Genre Memphisia Khalil, 1922, Neveu-Lemaire emend.}

Ce genre ne se distingue, d'après M. Khalil, du genre Pteridopharynx que par la présence d'un renflement vésiculeux autour de la tête et par l'existence d'une expansion à la côte dorsale externe. Mais ce dernier caractère me semble n'avoir qu'une valeur spécifique, car ce rameau de la dorsale externe existe ou non chez des espèces très voisines, ainsi que nous le verrons plus loin.

Diagnose. - Cylicostominæ à extrémité antérieure droite, chez lesquels un renflement vésiculeux bien développé entoure la tête. 
La bouche, terminale, est entourée d'une coronule externe, dont les lamelles, de longueur variable, ne font pas sailtie en avant. La cavité buccale est profonde et son diamètre est plus grand que sa hauteur; elle présente sur son plancher des dents chitineuses. Il existe une dépression en forme d'entonnoir à la naissance de l'œsophage qui est court et épais. La structure plumeuse de la cuticule œesophagienne est, comme dans le genre précédent, présente ou absente.

Mále. - La côte latérale postérieure porte une proéminence sur son bord postérieur et la côte dorsale externe présente ou non une branche dirigée en arrière. Les deux branches externes de la dorsale médiane sont fusionnées en une seule sauf à la pointe. Les spicules sont égaux et semblables et il existe une pièce accessoire.

Femelle. - La vulve est située immédiatement en avant de l'anus. Le vagin se dirige antérieurement, ainsi que les utérus qui sont parallèles; chacun d'eux est pourvu d'un ovéjecteur puissant.

TYPe du GenRe. - Memphisia memphisia Khalil, 1922, de l'intestin de l'éléphant d'Afrique, Loxodonta africana.

Memphisia rhinocerotis $n$. sp.

(Planche XIII)

Description. - Le corps est cylindroïde, un peu obtus aux extrémités, de couleur blanchâtre et possède une cuticule striée en travers. La bouche, terminale, est entourée de quatre papilles proéminentes, terminées par un petit prolongement en forme de bouton et limitée par une coronule externe à lames de longueur variable et convergentes. La capsule buccale est à peu près cylindrique. Un renflement vésiculeux annulaire entoure la tête au niveau des deux tiers postérieurs de la capsule buccale et est suivi de quatre expansions aliformes striées transversalement, ellesmêmes suivies d'expansions plus petites. L'œsophage est court, rétréci au niveau de l'anneau nerveux, situé aux environs de son tiers supérieur et renflé dans ses deux tiers postérieurs. L'intérieur de l'œsophage présente, dans sa portion antérieure, immédiatement au-dessus de lanneau nerveux, un organe particulier à structure plumeuse. L'extrémité postérieure est pourvue de valvules. Le pore excréteur se trouve à quelque distance de l'extrémité postérieure de l'œsophage. L'intestin présente des cellules bien marquées.

Mâle. - Le mâle a sensiblement le même diamètre dans toute sa longueur, sauf dans le dernier tiers, légèrement rétréci; les deux extrémités semblent tronquées. Le lobe dorsal de la bourse caudale est assez développé. La côte latérale postérieure porte une pe- 
tite proéminence sur son bord postérieur. Les côtes dorsales externes naissent isolément. La côte dorsale, épaisse à sa naissance, est disjointe jusque vers son tiers postérieur; elle donne naissance, vers son tiers antérieur, de chaque côté, à une branche qui ne présente une ébauche de division, que tout à fait à son extrémité ; puis ses deux parties se rejoignent sur une petite étendue par un pont, d'où partent deux branches terminales. Les spicules sont égaux et semblables, contournés et finement striés. Il existe une pièce accessoire.

Femelle. - La femelle, un peu plus longue que le mâle, a un diamètre sensiblement égal dans toute sa longueur, s'atténuant légèrement aux deux extrémités, qui paraissent tronquées, bien que l'extrémité postérieure porte une petite pointe. L'anus est situé à la naissance de cette pointe et la vulve est tout à fait voisine de l'anus. Dans le vagin, relativement court, débouchent deux ovéjecteurs parallèles, auxquels aboutissent deux utérus descendant parallèlement depuis leur origine. Les ovaires décrivent des circonvolutions, sans s'avancer très loin vers l'extrémité antérieure.

Les œufs contenus dans les utérus sont ovoïdes, à cuticule mince et ont subi un commencement de segmentation.

\section{DIMENSIONS DE MENPHISIA RHINOCEROTIS}

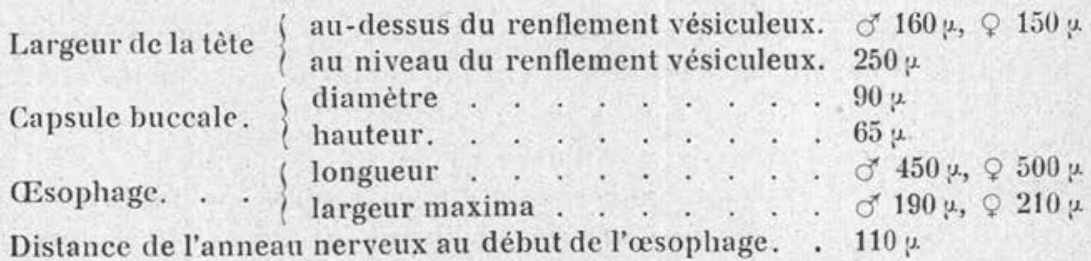

Màle

Longueur . . . . . . . . . . . . . . . . $13^{\mathrm{mm}}$

Largeur maxima . . . . . . . . . . . . . . $550 \mu$

Longueur de la côte dorsale . . . . . . . . . . $500 \mu$

Longueur des spicules . . . . . . . . . . . $850 \%$

Longueur du gorgeret . . . . . . . . . . $50 \mu$

Femelle

Longueur . . . . . . . . . . . . $18 \mathrm{~mm}$

Largeur maxima . . . . . . . . . . . . . . $700 \mu$

Distance de l'anus à l'extrémité postérieure . . . . . $650 \mu$

Distance de la vulve à l'extrémité postérieure . . . . 675u

Dimensions des œufs dans l'utérus . . . . . . . . $65 \mu \times 30 \mu$ 
Hôte. - Cette espèce se rencontre en assez grand nombre dans le gros intestin du rhinocéros d'Afrique, Rhinoceros bicornis L.

Localité. - Voisinage de la rivière Ousnée, affluent du fleuve Omo, dans la région du lac Rodolphe (Afrique orientale).

\section{Genre Henryella n. $g$.}

Ce genre est très voisin du genre Murshidia C. Lane, 1914; il s'en rapproche par la conformation de l'extrémité antérieure, mais s'en distingue par la disposition des côtes de la boursé caudale. Nous le dédions au professeur Henry, le savant helminthologiste de l'Eeole nationale vétérinaire d'Alfort.

Diagnose. - Cylicostominæ à extrémité antérieure droite. La tête est surmontée de quatre papilles submédianes très développées et proéminentes : une dorsale, une ventrale et deux latérales. La bouche est entourée d'une coronule externe à lamelles nombreuses et convergentes. La capsule buccale est cylindrö̈de. L'œesophage, assez court, est élargi en arrière de l'anneau nerveux. Sa partie postérieure est munie de valvules. Les cellules de l'intestin sont assez bien marquées.

Mále. - Le lobe dorsal de la bourse caudale du mâle est plus développé que les lobes latéraux. La partie moyenne des côtes latérales postérieures porte dorsalement une excroissance arrondie. La côte dorsale externe naît isolément. La dorsale médiane ne porte, de chaque côté, que deux branches, dont l'externe est à peine divisée et seulement tout à fait à son extrémité. Les spicules sont égaux et grêles ; il existe une pièce accessoire.

Femelle. - L'extrémité postérieure de la femelle se termine en pointe. La vulve est située un peu en avant de l'anus. Les utérus sont parallèles.

TYPE DU GENRE. - Henryella Raillieti n. g., n. sp. du gros intestin du rhinocéros africain, Rhinoceros bicornis.

Henryella Raillieti n. g., n. sp. (Planche XIV)

Description. - Le córps est cylindroïde, de couleur blanchâtre, à cuticule nettement striée transversalement. La tête est petite $t$ suivie d'un rétrécissement au niveau de la naissance de l'œsophage, puis te diamètre du corps augmente rapidement. Les quatre papilles submédianes sont très développées, longues et terminées par un bouton ovoïde. La bouche est entourée d'une coronule formée de 
lames convergentes. La capsule buccale est cylindrique. Les glandes céphaliques sont bien développées. L'œesophage est court, à peine resserré au niveau de l'anneau nerveux, situé un peu en arrière de son tiers antérieur. La partie terminale de l'œsophage est munie de valvules. Les cellules intestinales sont distinctes.

Mâle. - Le mâle est légèrement atténué à ses extrémités. Le lobe dorsal de la bourse caudale est plus développé que les lobes latéraux. La côte latérale postérieure présente dorsalement un diverticule arrondi. La côte dorsale médiane ne porte de chaque côté que deux branches, dont l'externe est à peine divisée à son extrémité. Les spicules sont égaux et grêles; il existe une pièce accessoire.

Femelle. - La femelle est un peu atténuée à ses extrémités, surtout à l'extrémité postérieure qui se termine par une longue pointe. La vulve est située à une petite distance de l'anus; le vagin est assez court ; les ovéjecteurs et les utérus sont parallèles ; les ovaires forment des anses, sans décrire de circonvolutions. Les œufs observés dans les ovéjecteurs ou les utérus sont en voie de segmentation.

Hôte. - Plusieurs exemplaires de cette espèce ont été rencontrés dans le gros intestin du rhinocéros d'Afrique, Rhinoceros bicornis $\mathrm{L}$.

Localité. - Voisinage de la rivière Ousnée, aftluent du fleuve Omo, dans la région du lac Rodolphe (Afrique orientale).

\section{Henryella Bozasi n. g., n. sp.}

(Planche XV)

Description. - Cette espèce est sensiblement plus grande que la précédente; son corps est cylindroïde, de couleur blanchâtre, avec une cuticule striée transversalement. La tête est moins nettement séparée du corps que chez $H$. Raillieti. Comme chez cette dernière espèce, les quatre papilles submédianes sont allongées et terminées par un bouton ovoïde. La bouche est entourée d'une coronule à lames convergentes, mais la capsule buccale est subglobuleuse au lieu d'être cylindrique. Les glandes céphaliques sont bien développées. L'œsophage est sensiblement plus long que chez les autres espèces du même genre ; son plus grand diamètre est situé vers le quart postérieur; il présente des valvules à la naissance de l'intestin, dont les cellules apparaissent distinctement.

Mâle. - Le mâle est très légèrement atténué à ses extrémités. Le lobe dorsal de la bourse caudale est beaucoup plus grand que 
chez $H$. Raillieti. La côte latérale postérieure porte dorsalement un diverticule irrégulièrement arrondi et volumineux. La côte dorsale externe est renflée par endroits. La dorsale médiane est longue et divisée en deux branches, dont l'externe est à peine divisée à sa pointe. Les spicules sont égaux et grêles et il existe une pièce accessoire.

Femelle. - La femelle, peu atténuée aux extrémités, l'est un peu plus à l'extrémité postérieure, qui se termine par une pointe relativement courte. La vulve est très voisine de l'anus ; elle en est beaucoup plus rapprochée que dans l'espèce précédente. Les ovéjecteurs et les utérus sont parallèles ; les ovaires forment quelques anses, mais ne déerivent point de circonvolutions. Les œufs contenus dans les utérus sont segmentés.

Nous dédions cette espèce à la mémoire du vicomte du Bourg de Bozas, chef de la Mission au cours de laquelle furent récoltés ces nématodes.

Hôte. - Nous avons trouvé un certain nombre d'individus de cette espèce dans le gros intestin du rhinocéros d'Afrique, Rhinoceros bicornis $\mathrm{L}$.

Localité. - Voisinage de la rivière Ousnée, affluent du fleuve Omo, dans la région du lac Rodolphe (Afrique orientale).

Henryella Zeltneri n. g., n. sp. (Planche XVI)

Description. - Le corps est cylindroïde, de couleur blanchàtre et les stries transversales de la cuticule sont peu marquées. La tête est petite, séparée du corps par un étranglement assez net au niveau de la naissance de l'œsophage. Les quatre papilles submédianes sont longues et terminées par un bouton ovoïde. Les papilles latérales sont volumineuses et présentent aussi un petit boutón terminal. La bouche est entourée d'une coronule externe à lames convergentes et l'on distingue nettement une coronule interne. La capsule buccale est cylindrique. Les glandes céphaliques sont biea développées. L'œsophage est court, à peine rétréci au niveau de l'anneau nerveux et sensiblement plus large dans sa partie postérieure que dans la région antérieure; il présente des valvules à sa partie terminale. Les cellules intestinales sont distinctes.

Mâle. - Le mâle est très peu atténué aux extrémités. Le lobe dorsal de la bourse caudale est plus grand que les lobes latéraux. La côte dorsale médiane porte deux branches; la branche externe est à peine divisée à son extrémité. Les spicules sont assez longs, grêles et obliquement striés; la pièce accessoire est petite. 
Femelle. - Je n'ai pas observé d'exemplaire femelle.

Cette espèce est dédiée à M. de Zeltner, membre de la Mission du Bourg de Bozas. Elle me paraît très voisine, bien que distincte, de l'espèce décrite par M. Khalil, sous le nom de Memphisia aziza, dans l'intestin de l'éléphant d'Afrique.

Hôte. - Ce nématode a été observé dans le gros intestin du rhinocéros d'Afrique, Rhinoceros bicornis $\mathrm{L}$.

Lochlité. - Voisinage de la rivière Ousnée, affluent du fleuve Omo, dans la région du lac Rodolphe (Afrique orientale).

\section{Henryella Didieri n. g., n. sp.}

(Planche XVI)

Description. - Le corps est cylindroïde, de couleur blanchâtre, avec des stries cuticulaires transversales peu marquées. La tête, petite, est suivie d'un rétrécissement au niveau de la naissance de l'œesophage. Les quatre papilles submédianes sont saillantes, mais moins grandes que chez les trois espèces précédentes et ne portent point de bouton terminal. Les papilles latérales sont très nettes. La bouche est entourée d'une coronule externe formée de lames convergentes. La capsule buccale est cylindrique. Les glandes céphaliques sont bien développées. L'œsophage est court, un peu rétréci au niveau de l'anneau nerveux et s'élargit dans son tiers postérieur; il présente des valvules à la naissance de l'intestin, dont les cellules sont assez distinctes.

Mâle. - Je n'ai pas rencontré d'exemplaires mâles.

Femelle. - La femelle est légèrement atténuée à ses extrémités, surtout à l'extrémité postérieure, terminée par une pointe effilée. La vulve est située un peu en avant de l'anus. Le vagin est court ; les ovéjecteurs et les utérus sont parallèles; les ovaires décrivent quelques sinuosités qui s'avancent jusque vers le tiers antérieur du corps. Les œufs contenus dans les utérus n'étaient point arrivés à maturité.

Nous dédions cette espèce à la mémoire de M. Didier, l'un des membres de la Mission du Bourg de Bozas.

Hôte. - Cette espèce habite le gros intestin du rhinocéros d'Afrique, Rhinoceros bicornis $\mathrm{L}$.

Localité. - Voisinage de la rivière Ousnée, affluent du fleuve Omo, dans la région du lac Rodolphe (Afrique orientale).

Nous donnons dans le tableau suivant les dimensions comparées des quatre espèces d'Henryella précédemment décrites. 
DIMENSIONS DES ESPĖCES DU GENRE HENRYELLA

\begin{tabular}{|c|c|c|c|c|}
\hline & H. Raillieti & H. Bozasi & $\begin{array}{c}\text { H. } \\
\text { Zeltneri }\end{array}$ & $\begin{array}{c}\text { H. } \\
\text { Didieri }\end{array}$ \\
\hline 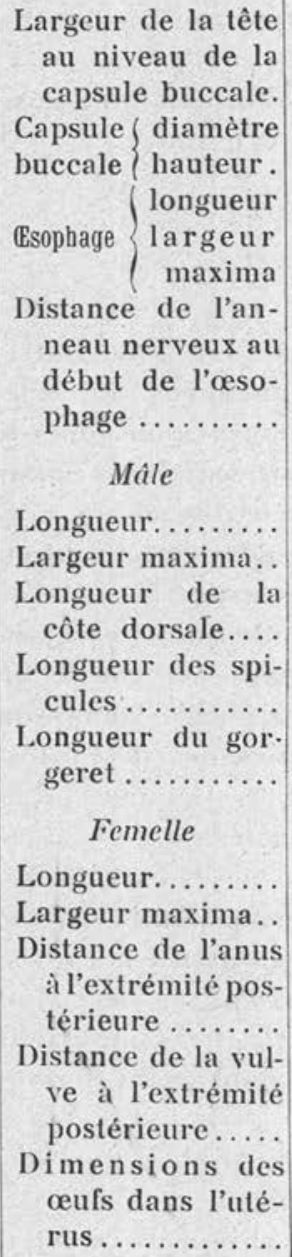 & $\begin{array}{c}\text { o } 110 \mu, \text { o } 100 \mu \\
40 \mu \\
50 \mu \\
\text { ơ } 380 \mu, \text { } 370 \mu \\
130 \mu\end{array}$ & $\begin{array}{c}110 \mu \\
\sigma^{\times} 60 \mu, \text { } 50 \mu \\
50 \mu \\
\sigma^{\times} 520 \mu, q 560 \mu \\
150 \mu \\
\sigma^{7} 120 \mu, q 150 \mu \\
15 \mathrm{~mm} . \\
650 \mu \\
700 \mu\end{array}$ & $\begin{array}{l}80 \% \\
11 \mathrm{~mm} . \\
500 \% \\
425 \% \\
760 \% \\
50 \%\end{array}$ & $\begin{array}{r}110 \mu \\
35 \mu \\
40 \mu \\
350 \mu \\
130 \mu\end{array}$ \\
\hline
\end{tabular}

Genre Buissonia n. g.

Ce genre se distingue du genre Henryella par le grand développement de la coronule externe qui fait saillie antérieurement et 
par l'absence ou l'atrophie des quatre papilles submédianes, si développées dans le genre précédent. Nous lui donnons ce nom en mémoire du $D^{r}$ Jean Buisson, jeune naturaliste, qui a étudié les infusoires parasites du rhinocéros africain et qu'une mort accidentelle au Congo belge a ravi prématurément à la science.

Diagnose, - Cylicostominæ à extrémité antérieure droite. La bouche est entourée par une coronule externe très développée, formée de nombreuses lames convergentes. Ces lames s'insèrent à leur base, à la face interne de la capsule buccale et se rapprochent les unes des autres par leur extrémité libre, ce qui donne à la cavité buccale l'aspect d'un entonnoir renversé. Il existe en outre une coronule interne plus ou moins marquée. La capsule buccale est à peu près cylindrique. L'œsophage, relativement court, atteint en arrière de l'anneau nerveux, sa largeur maxima; sa partie postérieure est munie de valvules.

Mále. - Le lobe dorsal de la bourse caudale du mâle est plus développé que les lobes latéraux. La partie moyenne des côtes latérales postérieures porte dorsalement une excroissance arrondie. Les côtes dorsales externes naissent isolément et portent souvent aussi dorsalement une excroissance assez longue vers leur partie moyenne. La côte dorsale est divisée dans sa portion médiane presque jusqu'à l'endroit où elle se bifurque, point au niveau duquel existe une sorte de pont; elle donne naissance à deux branches, dont l'externe est à peine divisée à son extrémité. La côte dorsale et surtout ses ramifications internes présentent fréquemment des diverticules plus ou moins longs. Les spicules sont égaux et grêles ; il existe une pièce accessoire.

Femelle. - L'extrémité postérieure de la femelle se termine en pointe. La vulve est plus ou moins rapprochée de l'anus. Les utérus sont parallèles.

TYPE DU GENRE. - Buissonia rhinocerotis n. g., n. sp., du gros intestin du rhinocéros africain, Rhinoceros bicornis.

\section{Buissonia rhinocerotis n. g., n. sp.}

(Planche XVII)

Description. - Le corps est cylindroïde, de couleur blanchâtre, à cuticule striẻe transversalement. La tête est petite et, après un léger rétrécissement situé un peu en árrière de la naissance de l'œsophage, le diamètre du corps augmente rapidement pour atteindre son maximum dans la région moyenne, puis il diminue gra- 
duellement à mesure qu'on se rapproche de l'extrémité postérieure. Les papilles submédianes sont peu développées et présentent un petit bouton terminal. La bouche est presque fermée par les lames convergentes de la coronule externe. La coronule interne suit le bord antérieur de la capsule buccale ; celle-ci est cylindrique. Les glandes céphaliques sont assez développées. L'œsophage est court, moins large en avant de l'anneau nerveux qu'en arrière. L'anneau nerveux est situé un peu en arrière du tiers antérieur de l'œsophage, qui présente son plus grand diamètre vers son tiers postérieur. Il existe des valvules à la naissance de l'intestin. Les cellules intestinales ne sont pas distinctes, tout au moins dans la partie qui est voisine de l'œsophage.

Mále. - Le mâle est légèrement atténué aux deux extrémités. Le lobe dorsal de la bourse caudale est plus développé que les lobes latéraux.

La côte latérale postérieure présente dorsalement un diverticule arrondi ; la côte dorsale externe porte, également dorsa-

- lement, un diverticule mince et allongé. La côte dorsale médiane, disjointe depuis sa base presque jusqu'au niveau de sa bifurcation, donne naissance vers son tiers antérieur, de chaque côté, à une branche externe, à peine divisée à son extrémité ; la côte dorsale porte, un peu avant sa bifurcation, de petits diverticules allongés, qui existent aussi, mais un peu moins développés du côté externe de chacune des branches de la bifurcation. Les spicules sont égaux, assez longs, grêles et en partie striés obliquement. II existe une pièce accessoire.

Femelle. - La femelle est à peine atténuée à ses extrémités ; l'extrémité postérieure est terminée en pointe. L'anus est situé à la naissance de la queue pointue et la vulve se trouve à peu de distance en avant de l'orifice anal. Le vagin, de longueur moyenne, reçoit deux ovéjecteurs qui cheminent parallèlement, ainsi que les utérus qui y aboutissent.

Les ovaires rejoignent les utérus un peu en avant du milieu du corps et forment des anses qui s'avancent jusqu'au niveau du tiers antérieur. Les ovéjecteurs et les utérus renferment des œufs ovoïdes, à cuticule mince, ayant déjà subi un commencement de segmentation.

Hôte. - Plusieurs exemplaires de cette espèce ont été trouvés dans le gros intestin du rhinocéros d'Afrique, Rhinoceros bicor. nis $\mathrm{L}$.

Localité. - Voisinage de la rivière Ousnée, affluent du fleuve Omo, dans la région du lac Rodolphe (Afrique orientale). 


\section{Buissonia africana n. g., n. sp.}

(Planches XVIII et XIX)

Description. - Le corps est cylindroïde, légèrement atténué aux deux extrémités, de couleur blanchâtre, avec une cuticule striée transversalement. La tête, petite, est moins brusquement séparée du corps que chez B. rhinocerotis. La bouche présente la même disposition que chez cette dernière espèce, avec une coronule externe et une coronule interne bien développées. Il existe deux petites papilles latérales; la capsule buccale est cylindrique. Les glandes céphaliques sont bien développées. L'œsophage est court, un peu moins large en avant de l'anneau nerveux qu'en arrière de celui-ci; son plus grand diamètre se trouve au niveau du tiers postérieur. Il existe des valvules à la terminaison de l'œsophage. Les cellules intestinales sont assez bien indiquées.

Mále. - Le mâle est à peine atténué aux extrémités. Le lobe dorsal de la bourse caudale est plus développé que les lobes latéraux. La côte latérale postérieure possède dorsalement un diverticule arrondi, mais la côte dorsale externe ne présente aucun diverticule. La côte dorsale médiane est disjointe depuis sa base jusqu'à sa bifurcation ; sa branche externe est dédoublée à l'extrémité seulement.

Sur un exemplaire, j'ai constaté le dédoublement complet de la branche externe droite, anomalie qui forme le terme de passage avec la disposition que l'on observe dans les genres Murshidia et Cylicostomum.

La terminaison de la côte dorsale et ses branches internes présentent des dentelures formées par de courts diverticules. Les spicules sont égaux, assez longs, grêles et striés obliquement. Il existe une pièce accessoire.

Femelle. - La femelle est légèrement atténuée à ses extrémités. L'extrémité postérieure est terminée en pointe à peine recourbée ventralement. La vulve est située tout contre l'anus. Le vagin est assez court; les ovéjecteurs et les utérus sont parallèles. Les ovaires, à peine contournés, forment. une anse qui n'atteint pas le tiers antérieur du corps. Les œufs ont subi un début de segmentation.

Hôte. - Cette espèce a été trouvée dans le gros intestin du rhinocéros d'Afrique, Rhinoceros bicornis L.

Localité. - Voisinage de la rivière Ousnée, affluent du fleuve Omo, dans la région du lac Rodolphe (Afrique orientale). 


\section{Buissonia longibursa n. g., n. sp. (Planche XIX)}

Description. - Le corps est cylindroïde, un peu atténué aux extrémités et de couleur blanchâtre; la cuticule est striée transversalement. La tête, contrairement à ce qui a lieu chez les deux espèces précédentes, est à peine distincte et se continue sans ligne de démarcation avec le reste du corps, mais la bouche est conformée comme chez ces dernières et possède une coronule externe affectant la même disposition. La coronule interne est moins nette. La capsule buccale est cylindrique. Les glandes céphaliques sont assez développées. L'œsophage, court, est un peu plus étroit dans son tiers antérieur, jusqu'à l'anneau nerveux, qu'en arrière de celui-ci et présente son plus grand diamètre vers son tiers postérieur.

Il existe des valvules dans sa position terminale. Les cellules intestinales sont peu distinctes dans la partie de l'intestin voisine de l'œsophage.

Mâle. - Le mâle est à peine atténué aux extrémités qui, à l'œil nu, paraissent tronquées. Le lobe dorsal de la bourse caudale est très développé, beaucoup plus que chez les deux espèces précédemment décrites, ce qui permet de distinguer cette espèce des deux autres. Les côtes dorsales externes portent vers leur milieu un long diverticule. La côte dorsale médiane est disjointe jusqu'à sa bifurcation; ses branches externes sont dédoublées tout à fait à l'extrémité.

Les spicules sont égaux, assez longs, grêles et striés obliquement. La pièce accessoire est petite.

Femelle. - Je n'ai pas rencontré d'exemplaires femelles.

Hôte. - Ce strongle a été trouvé dans le gros intestin du rhinocéros d'Afrique, Rhinoceros bicornis L.

Localité. - Voisinage de la rivière Ousnée, aflluent du fleuve Omo, dans la région du lac Rodolphe (Afrique orientale).

De même que nous avons donné précédemment sous forme de tableau les dimensions des différentes espèces du genre Henryella, nous donnons ici, dans le tableau suivant, les dimensions comparées des trois espèces du genre Buissonia que nous venons de décrire. 
Dimensions DES ESPĖCES DU GENRE BUISSONIA

\begin{tabular}{|c|c|c|c|}
\hline & B. rhinocerotis & B. africana & $\begin{array}{c}\text { B. } \\
\text { longibursa }\end{array}$ \\
\hline 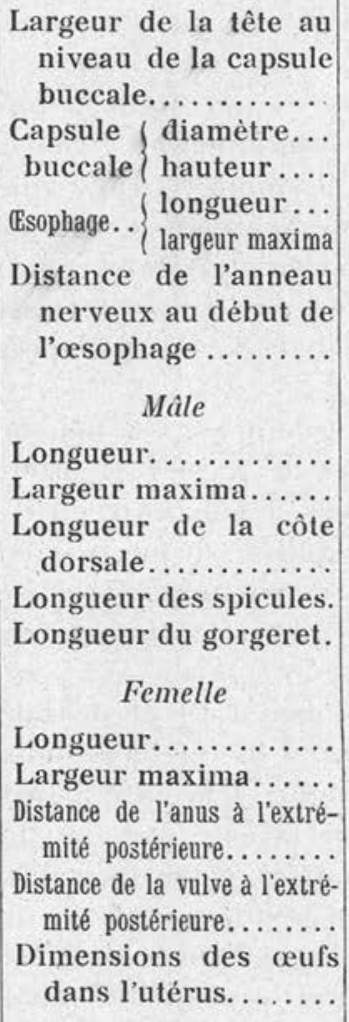 & $\begin{array}{c}100 \mu \\
40 \mu \\
40 \mu \\
350 \mu \\
\sigma^{\times} 170 \mu, \circ 150 \mu\end{array}$ & $\begin{array}{c}90 \mu \\
\sigma^{7} 30 \mu, \text { o } 40 \mu \\
40 \mu \\
\text { o } 400 \mu, \text { o } 570 \\
\sigma^{7} 130 \mu, \text { o } 170 \mu \\
\sigma^{7} 60 \mu, \text { o } 140 \mu\end{array}$ & $\begin{array}{r}16 \mathrm{~mm} . \\
620 \mu \\
700 \mu \\
730 \mu \\
50 \mu\end{array}$ \\
\hline
\end{tabular}

\section{Genre Kiluluma Skrjabin, 1916}

Le genre Kiluluma a été créé par Skrjabin, en 1916, pour un strongle du rhinocéros africain, Rhinoceros bicornis, strongle, qui avait été décrit, en 1907, par von Linstow, sous le nom de Dele. trocephalus stylosus.

Or Skrjabin, en étudiant des spécimens de ce nématode, faisant partie des collections du Musée de l'Académie impériale des Sciences de Petrograd, constata, chez le parasite du rhinocéros, des caractères qui le différenciaient nettement du genre Deletrocephalus, 
genre ayant pour type un Strongylidæ du nandou, Rhea americana, appelé $D$. dimidiatus Diesing, 1861. Railliet et Leiper pensent également que le strongle du rhinocéros ne doit pas être placé dans le même genre que le parasite du nandou.

Le nom de Kiluluma, adopté par Skrjabin, est celui de la localité, où ont étẻ tués les deux rhinocéros, qui hébergeaient les nématodes en question.

Parmi les nombreux strongles du rhinocéros que nous avons examinés, nous n'avons pas retrouvé de représentants de ce genre, ce qui tient peut-être à sa localisation dans l'estomac, tandis que nos exemplaires provenaient du gros intestin. Néanmoins, comme Kiluluma stylosa est le seul strongle du rhinocéros africain connu jusqu'ici, nous croyons intéressant de donner, en terminant cet article, la diagnose du genre et la description de l'espèce d'après Skrjabin.

Diagnose. - Cylicostominx de moyenne grandeur. La bouche est entourée de quatre lèvres périphériques et de six lèvres internes; les externes portant des papilles renflées à leur extrémité ; les internes étant surmontées de quatre papilles terminées en pointe. L'œsophage est renflé en massue dans sa partie postérieure. Les papilles cervicales ont la forme d'appendices terminés en pointe.

Mâle. - La bourse caudale du mâle est trilobée. La côte dorsale médiane, bifurquée à son extrémité, porte près de sa base deux côtes assez courtes, une de chaque côté. Chaque lobe latéral est soutenu par sept côtes qui sont, en partant de la côte dorsale : la côte dorsale externe qui naît isolément ; les trois côtes latérales, la latérale médiane et la latérale externe étant réunies sauf à leur extrémité ; les deux côtes ventrales soudées ; la septième côte n'est autre qu'une papille prébursale très développée. Les spicules sont égaux et spiralés.

Femelle. - La vulve est proche de l'anus.

Type du Genre. - Kiluluma stylosa (von Linstow, 1907), de l'estomac du rhinocéros africain, Rhinoceros bicornis.

Kiluluma stylosa (von Linstow, 1907)

(Planche XX)

Synonymie. - Deletrocephalus stylosus, von Linstow, 1907 ; Kiluluma stylosa, Skrjabin, 1916.

Description. - Le corps est cylindrö̈de, atténué aux extrémités et de couleur brune. L'extrémité céphalique présente une disposi- 
tion complexe, la bouche étant entourée d'une série de lèvres externes et internes. Des quatre lèvres externes, deux sont dorsales et deux ventrales, chacune d'elles étant pourvue d'une papille renflée à son extrémité libre. En dedans de ces quatre lèvres périphériques, se trouvent six lèvres internes, dont deux dorsales, deux ventrales et deux latérales. Les lèvres internes latérales ne portent point d'appendices, tandis que les lèvres internes dorsales et ventrales sont surmontées d'une papille terminée en pointe. L'œsophage est court et renflé en massue à sa partie postérieure. De chaque côté du corps, aussi bien chez le mâle que chez la femelle, la cuticule forme de larges anneaux qui vont en diminuant jusqu'à l'extrémité caudale. A quelque distance de l'extrémité céphalique se trouvent, dans les deux sexes, des papilles cervicales, formées d'une portion basale et terminées en pointe.

Mále. - La bourse caudale du mâle est formée de trois lobes ; les lobes latéraux sont divisés vers le milieu par une petite rainure. Les lobes de la bourse caudale sont soutenus par un système de côtes assez particulier et complexe. La côte dorsale médiane a son extrémité bifurquée et donne naissance, près de sa base, à deux côtes assez courtes, une de chaque côté. Chaque lobe latéral présente sept côtes qui sont, en partant de la côte dorsale : une côte dorsale externe qui naît isolément ; trois côtes latérales, la médiane et l'externe étant réunies sauf à leur extrémité ; deux côtes ventrales soudées et une septième côte, qui n'est, en réalité, qu'une papille prébursale ayant pris un grand développement. Les spicules présentent une structure particulière : striés transversalement sur la plus grande partie de leur longueur, ils sont contournés en spirale à leur extrémité, en forme de bêche. Il n'y a pas de pièce accessoire.

Femelle. - La femelle est terminée en pointe arrondie à l'extrémité. La vulve est très rapprochée de l'anus. Le vagin est très musclé.

DIMENSIONS DE KILELUMA STYLOSA

(D’après Skrjabin)

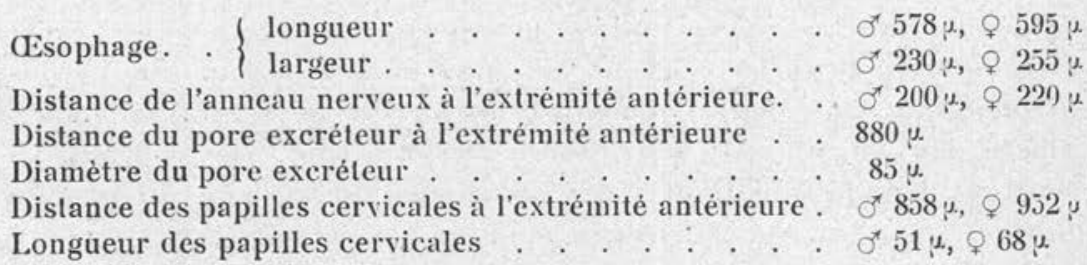


Mále

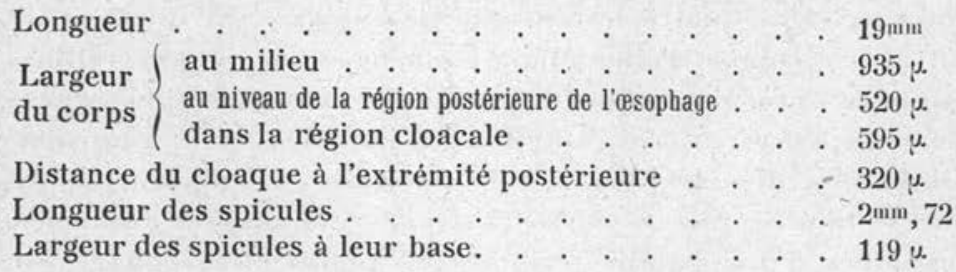

Femelle

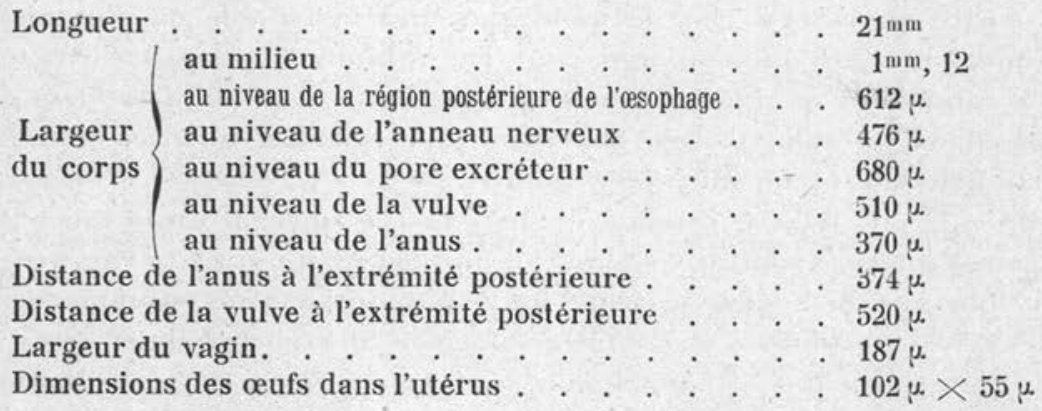

Hôte. - Cette espèce a été trouvẻe dans l'estomac du rhinocéros d'Afrique, Rhinoceros bicornis L.

Localité. - Bords de la rivière Tana, à Kiluluma (Afrique orientale).

\section{RÉSUMÉ}

Nous décrivons dans cette note treize espèces nouvelles de Strongylidx, provenant du gros intestin du rhinocéros d'Afrique, Rhinoceros bicornis L. et recueillies en Afrique orientale par le professeur E. Brumpt, au cours de la Mission du Bourg de Bozas.

Quatre de ces espèces appartiennent à des genres connus, ce sont: Quilonia rhinocerotis n. sp., parmi les Strongylinæ; Grammocephalus intermedius n. sp., parmi les Bunostominæ; Pteridopharynx omoensis n. sp. et Memphisia rhinocerotis n. sp., parmi les Cylicostominæ.

Les neuf autres espèces ont été groupées en quatre genres. L'une, Paraquilonia Brumpti n. g., n. sp., appartient à la sous-famille des Strongylinæ; une autre, Khalilia rhinocerotis n. g., n. sp., a été placée dans la nouvelle sous-famille des Amirinæ; les sept autres rentrent dans la sous-famille des Cylicostominæ, ce sont : Henryella Raillieti n. g., n. sp., H: Bozasi n. g., n. sp., H. Zeltneri n. g,, n, 
sp., H. Didieri n. g., n. isp., Buissonia rhinocerotis n. g., n. sp., $B$. africana n. g., n. sp., et B. longibursa n. g., n. sp.

En terminant, nous donnons la description, d'après Skrjabin, du seul strongle décrit jusqu'ici chez le rhinocéros africain, Kiluluma stylosa (von Linstow, 1907), qui nous paraît aussi devoir être rangé parmi les Cylicostominæ.

\section{BIBLIOGRAPHIE}

Baylis (H. A.). - A new Species of the Nematode genus Crossocephalus from the Rhinoceros. Ann. and Mag. of Natural History, série 9, IV, 1919, p. 94-98.

Evans (G. H.) et Rennie (T.), - Notes on some Parasites in Burmah III. A few common Parasites of Elephants. Journ. Trop. Vet. Science, Calculta, III, 1910, p. $240-256$.

IHLE (J. E. W.). - Ueber Ancylostoma perniciosum v. Linstow und die Strongyliden des Elephanten. Bijdr. tot de Dierk., Afl. 21, 1919, p. 97.

- Notiz zu meinem Aufsatz « Ueber Ancylosloma perniciosum v. Linstow und die Strongyliden des Elephanten ». Centralbl. für Bakt. und Paras., Orig., LXXXIII, 1919, p. 550.

KHaLiL (M.). - A preliminary Note on some new Nematode parasites from the Elephant. Annals and Magazine of Natural History, série 9, IX, férrier 1922, p. 212-216.

- A Revision of the Nemalode parasites of Elephants, with a description of four new species. Proceedings of the Zoological Sociely of London, juin 1922.

LaNe (C.). - Bursate Nematodes from the Indian Elephant. The Indian Journ. of med. Research, II, 1914-1915, p. 380-398.

- A further note on Bursate Nematodes from the Indian Elephant. The Indian Journ. of med. Research, III, 1915-1916, p. 105-108.

- Some Bursate Nematodęs from Indian and African Elephants. The Indian Journ. of med. Research, IX, 1921-1922, p. 163-173.

Leiper (R. T.). - Nematodes Kilimandjaro, 1909, p. 26.

Lisstow (O. von). - Nematoden aus dem Königlichen Museum in Berlin. Milleil. aus dem Zoolog. Mus. Berlin, III, 1907, p. 254.

Railliet (A.), Henry (A.) et Bauche (J.). - Sur les Helminthes de l'Eléphant d'Asie. II. Nématodes. A. Bull. Soc. pathol. exot., VII, 1914, p. 129-132.

- Sur les Helminthes de l'Eléphant d'Asie. IIl. Nematodes. B. Bull. Soc. palhol. exot., VII, 1914, p. 206-210.

- Sur les Helminthes de l'Eléphant d'Asie (Note complémentaire). Bull. Soc. pathol. exot., VIII, 1915, p. 117-119.

SkrJabin (K. I,). - Parasitic Trematodes and Nematodes collected by the expeditions of Prof. V. Dogiel and I. Sokolow in British Easl Africa, 1916, p. 151154 (en russe et en anglais).

Laboratoire de Parasilotogie de la Facullé de médecine de Paris. 


\section{EXPLICATION DES PLANCHES}

\section{Planche IX}

Quilonia rhinocerotis n. sp. - 1. Mâle et femelle, grandeur naturelle. 2. Extrémité antérieure du mâle, face latérale gauche. 3 . Tête du mâle vue de la même manière à un plus fort grossissement. 4. Bourse caudale du mâle vue obliquement du côté gauche. 5. Spicules et pièce accessoire. 6. Extrémité postérieure de la fémelle, face latérale droite. 7 . Région vulvaire de la femelle, face latérale gauche.

\section{Planche $\mathrm{X}$}

Paraquilonia Brumpti n. g., n. sp. - 1. Mâle et femelle, grandeur naturelle. 2. Extrémité antérieure du mâle vue par la face ventrale èt un peu obliquement. 3. Tête du mâle vue de la même manière à un plus fort grossissement. 4. Tête du mâle, face latérale droite. 5. Extrémité postérieure du mâle, face ventrale. 6. Bourse caudale du mâle vue du côté gauche. 7. Extrémité postérieure de la femelle vue du côté gauche. 8 . Région vulvaire de la femelle vue du côté gauche.

\section{Planche XI}

Grammocephalus intermedius n. sp. - 1. Mâle et femelle, grandeur naturelle. 2. Extrémité antérieure de la femelle. 3. Tête de la femelle vue à un plus fort grossissement. 4. Extrémité postérieure du mâle vue du côté droit. 5. Bourse caudale vue du côté droit. 6. Spicule droit très grossi vu latéralement.

\section{Planche XII}

Khalilia rhinocerotis n. g., n. sp. - 1. Mâle, grandeur naturelle. 2. Extrémité antérieure du mâle, face latérale droite. 3. Tête du mâle vue à un plus fort grossissement, face latérale gauche. 4. Détails de la striation cuticulaire. 5. Extrémité postérieure du mâle vue un peu obliquement par la face ventrale (la papille prébursale n'a pas été représentée).

Pteridopharynx omoensis n. sp. - 6. Femelle, grandeur naturelle. 7. Extrémitéantérieure de la femelle, face latérale gauche. 8 . Tête de la femelle vue de la même manière à un plus fort grossissement. 9. Extrémité postérieure de la femelle vue du côté gauche.

\section{Planche XIII}

Memphisia rhinocerotis n. sp. - 1. Mâle et femelle, grandeur naturelle. 2. Extrémité antérieure du mâle, face ventrale. 3 . Tête du mâle vue de la même manière à un plus fort grossissement. 4. Ornementation de l'œsophage. 5. Bourse caudale du mâle, face ventrale. 6 . Bourse caudale sue du côté gauche. 7 . Spicules et pièce accessoire. 8 . Extrémité postérieure de la femelle vue un peu obliquement du côté droit. 9. Extrémité postérieure de la femelle plus grossie et vue du côté gauche. 


\section{Planche XIV}

Henryella Raillieti n. g., n. sp. - 1. Mâle et femelle, grandeur naturelle. 2. Extrémité antérieure du mâle, face latérale droite. 3 . Tête du mâle vue de la même manière à uu plus fort grossissement. 4. Bourse caudale du mâle, face latérale droite. 5. Extrémité postérieure de la femelle vue un peu obliquement par la face ventrale. 6 . Extrémité postérieure de la femclle plus grossie et vue du côté gauche.

\section{Planche XV}

Henryella Bozasi n. g., n. sp. - 1. Mâle et femelle, grandeur naturelle. 2. Extrémité antérieure de la femelle, face latérale droite. 3 . Tête du mâle vue à un plus fort grossissement, face latérale droite. 4. Bourse caudale du mâle vue obliquement du côté droit. 5. Extrémité postérieure de la femelle vue du côté droit.

\section{Planche XVI}

Henryella Zeltneri n. g., n. sp. - 1. Mâle, grandeur naturelle. 2. Extrémité antérieure du mâle, face dorsale. 3. Tête du mâle vue de la même manière à un plus fort grossissement. 4. Extrémité postérieure du mâle, face dorsale.

Henryella Didieri n. g., n. sp. - 5. Femelle, grandeur naturelle. 6, Extrémité antérieure de la femelle, face latérale droite. 7 . Tête de la femelle vue de la même manière à un plus fort grossissement. 8. Extrémité postérieure de la femelle vue du côté droit.

\section{Planche XVII}

Buissonia rhinocerolis n. g., n. sp. - 1. Mâle et femelle, grandeur naturelle. 2. Extrémité antérieure de la femelle, face latérale gauche. 3. Tête de la femelle vue de la même manière à un plus fort grossissement. 4. Extrémité postérieure du mâle vue par la face ventrale. 5. Bourse caudale du mâle vue du côté gauche. 6. Extrémité postérieure de la femelle vue un peu obliquement du côtê gauche.

\section{Planche XVIII}

Buissonia africana n. g., n. sp. - 1. Mâle et femelle, grandeur naturelle. 2. Extrémité antérieure du mâle vue par la face ventrale. 3 . Tête du mâle vue de la même manière à un plus fort grossissement. 4. Spicules. 5. Extrémité postérieure de la femelle vue du côté gauche. 6 . Extrémité postérieure de la femelle vue du même côté à un plus fort grossissement. 


\section{Planche XIX}

Buissonia africana n. g., n. sp. - 1. Extrémité postérieure du mâle vue par la face ventrale. 2. Bourse caudale anormale vue par la face ventrale et dans laquelle la branche externe de droite de la côte dorsale médiane est divisée dans toute sa longueur.

Buissonia longibursa n. g., n. sp. - 3. Mâle, grandeur naturelle. 4. Extrémité antérieure du mâle vue par la face ventrale. 5. Tête du mâle vue de la même manière à un plus fort grossissement. 6 . Bourse caudale du mâle vue par la face dorsale.

\section{Planche XX}

(D'après Skrjabin)

Kiluluma stylosa. - 1. Extrémité antérieure du mâle; $e$, pore excréteur ; $c$, papilles cervicales. 2. Pore excréteur très grossi. 3. Extrémité céphalique avec lèvres et papilles. 4. Bourse caudale du mâle. 5 . Spicule, spiralé à sa pointe. 6 . Détails de structure du spicule. 7. Extrémité postérieure de la femelle, face ventrale. 
Tome II. PL. IX.

Mémoire Neveu-Lemaire

2.
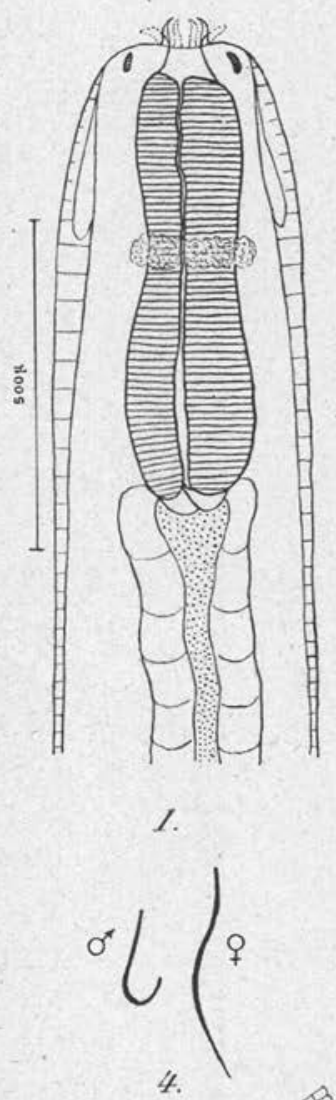

3.
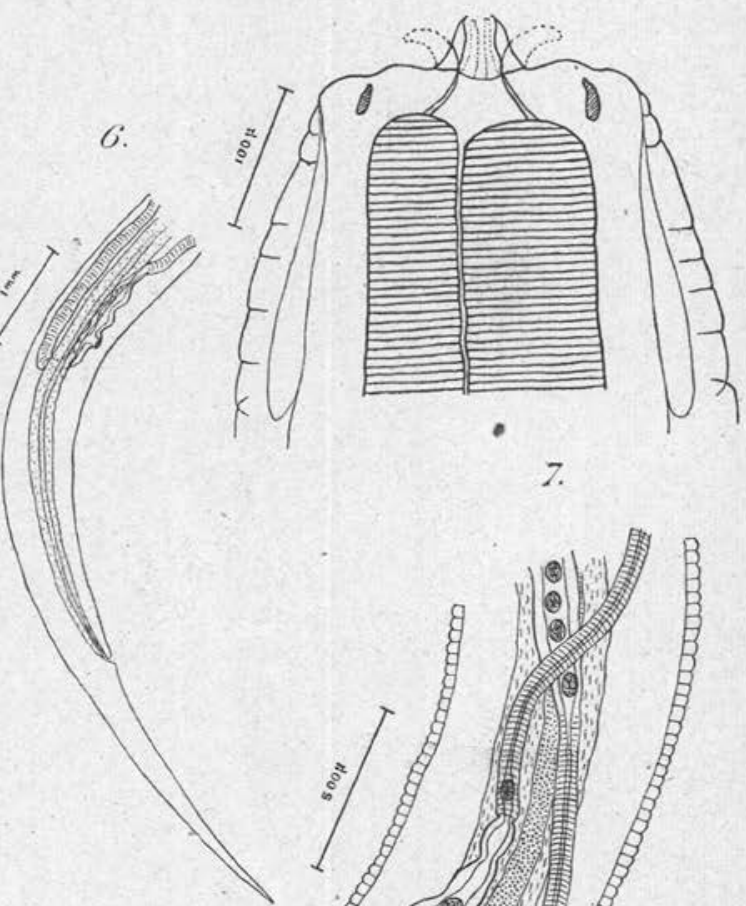

5.

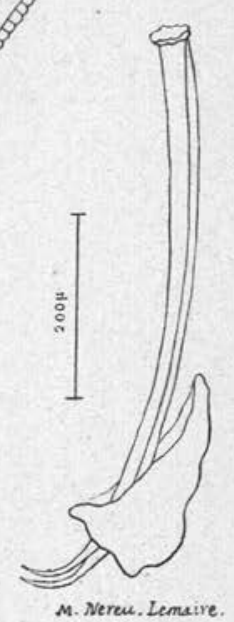

QUILONIA RHINOCEROTIS n. sp. 

2.

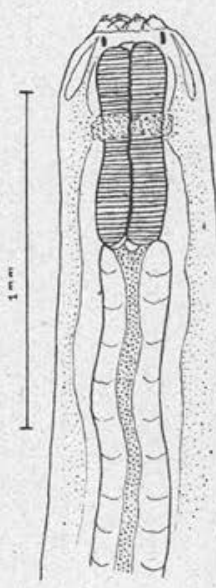

5.
7.
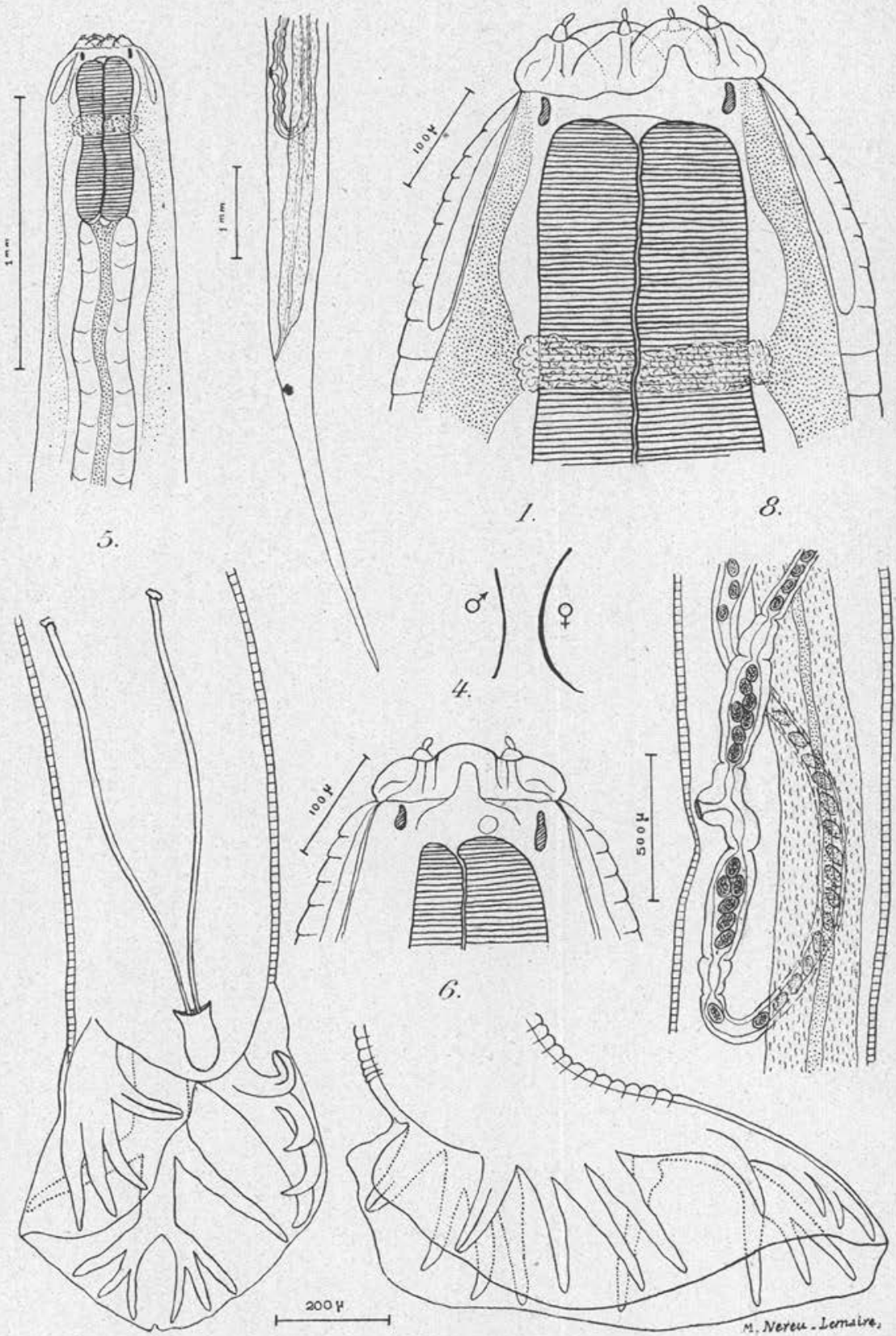

Paraquilonia Brumpti n. g., n. sp. 



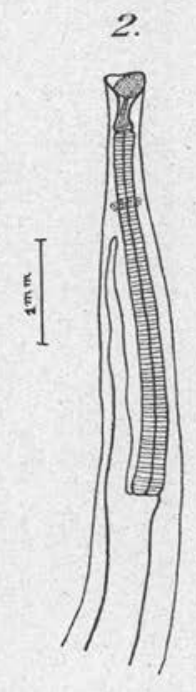

3.

6.
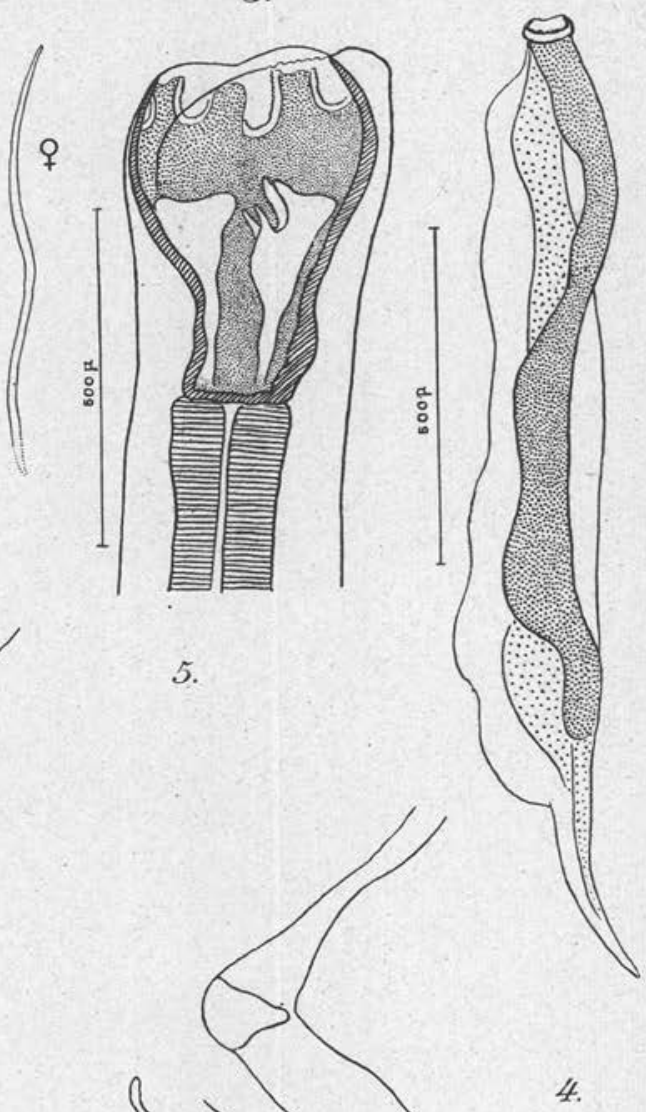

5.
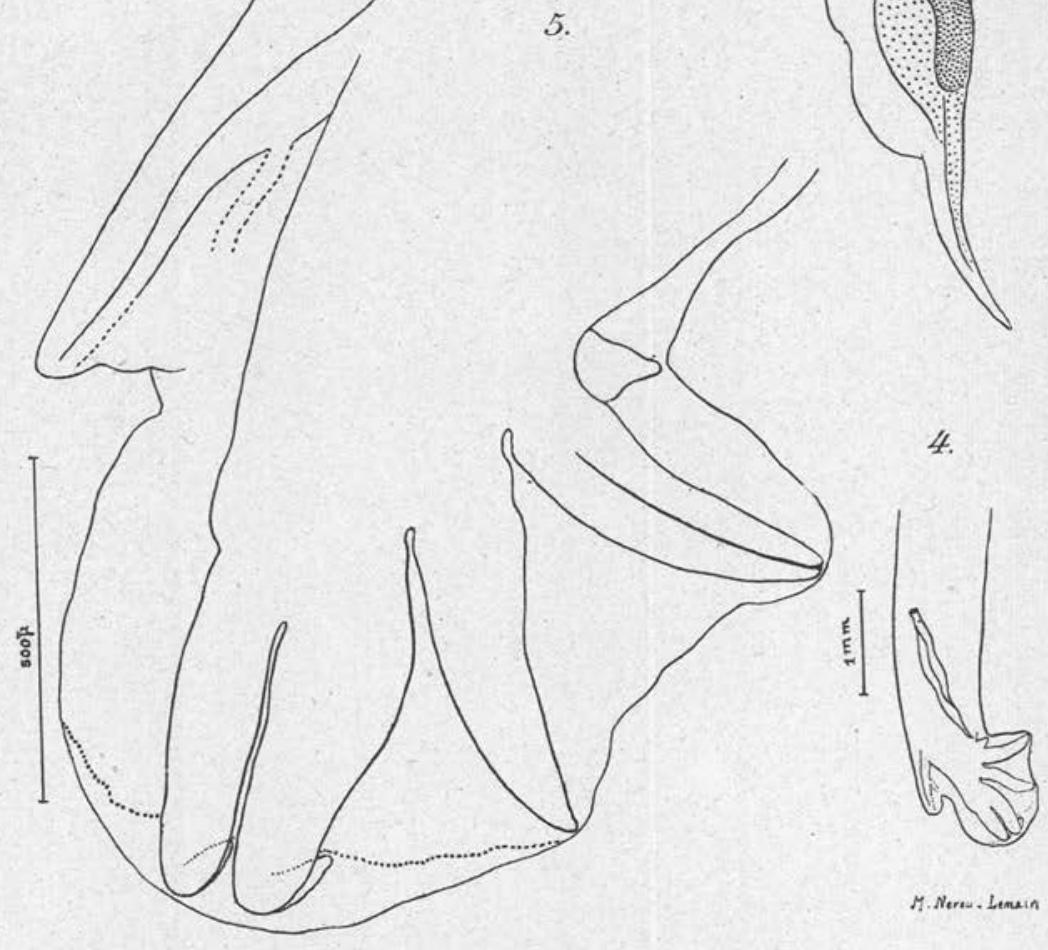

Grammocephalus intermedius n. sp. 

Tome II. PL. XII.

Mémoire Neveu-Lemaire

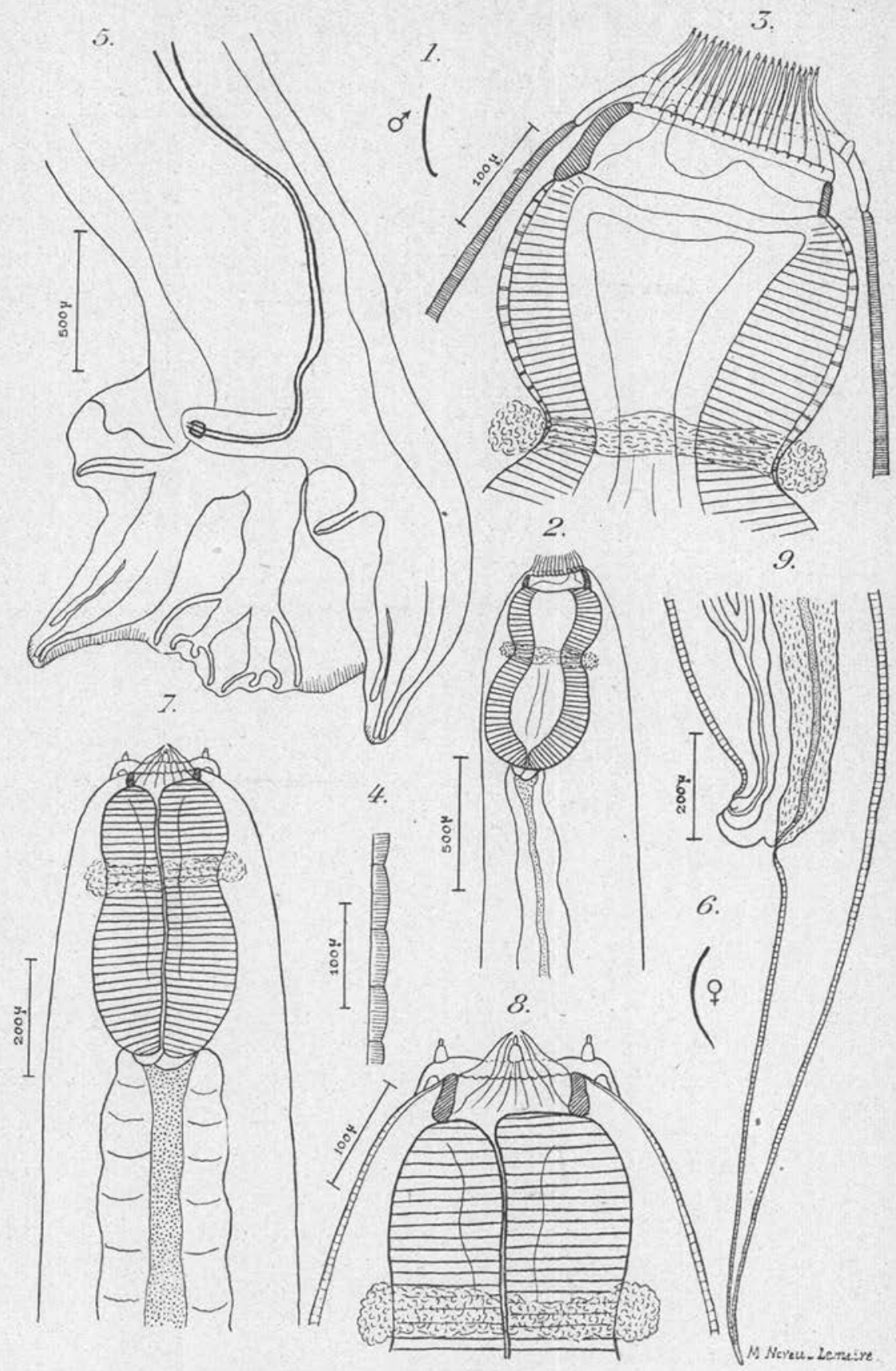

Khalilia RHiNocerotis n. g., n. sp. (1 à 5 )

Pteridopharynx omoensis n. sp. (6 à 9$)$ 



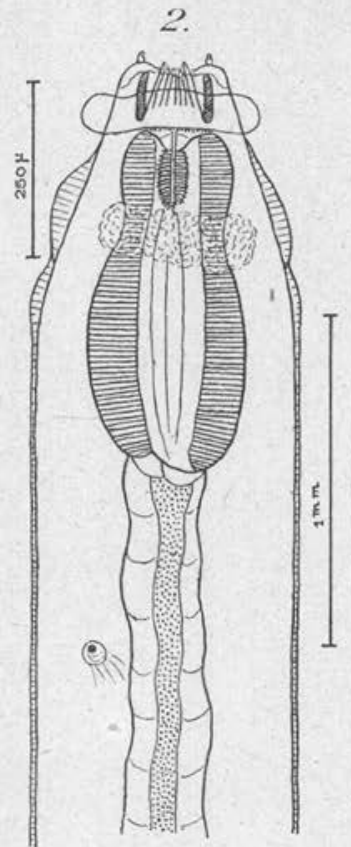

8. 3.

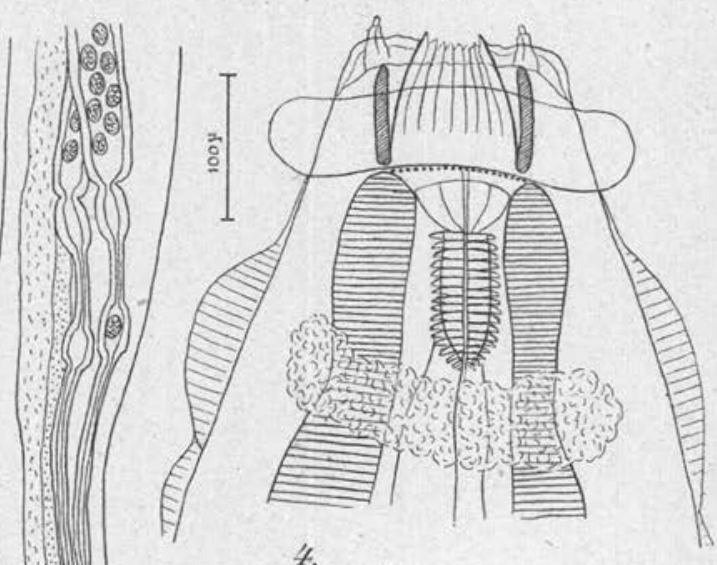

4.

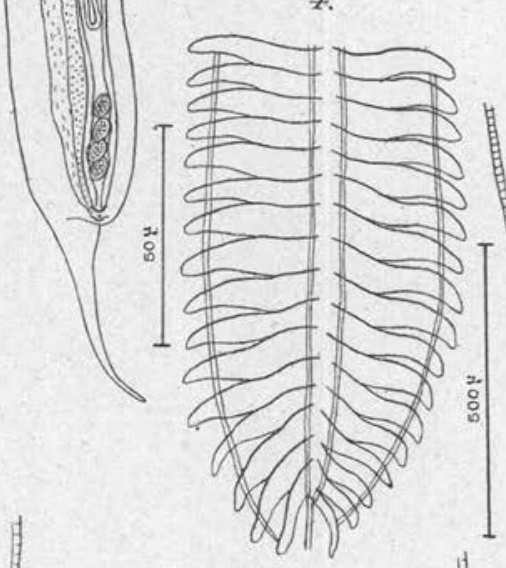

9.
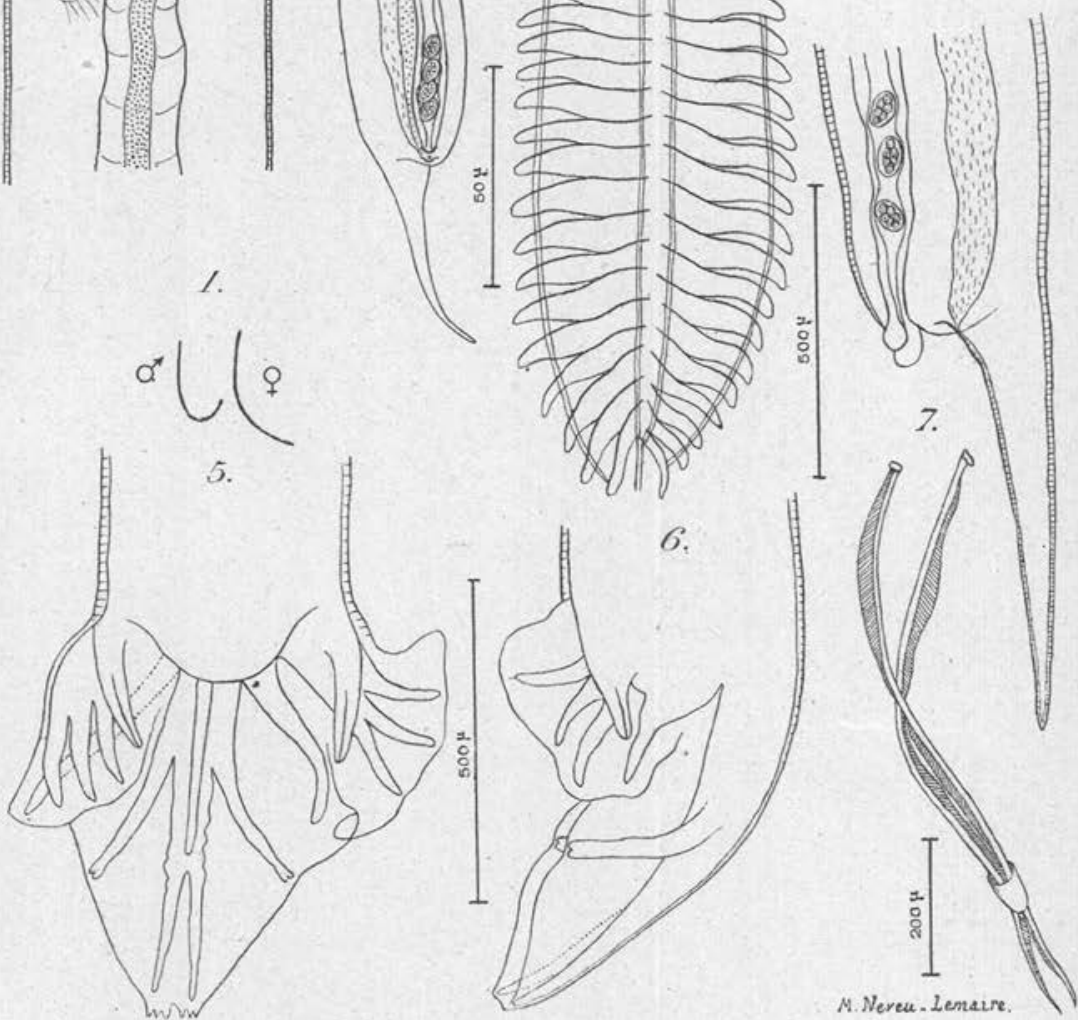

MEMPHISIA RHINOCEROTIS n. sp. 

ANNALES DE PARASITOLOGIE

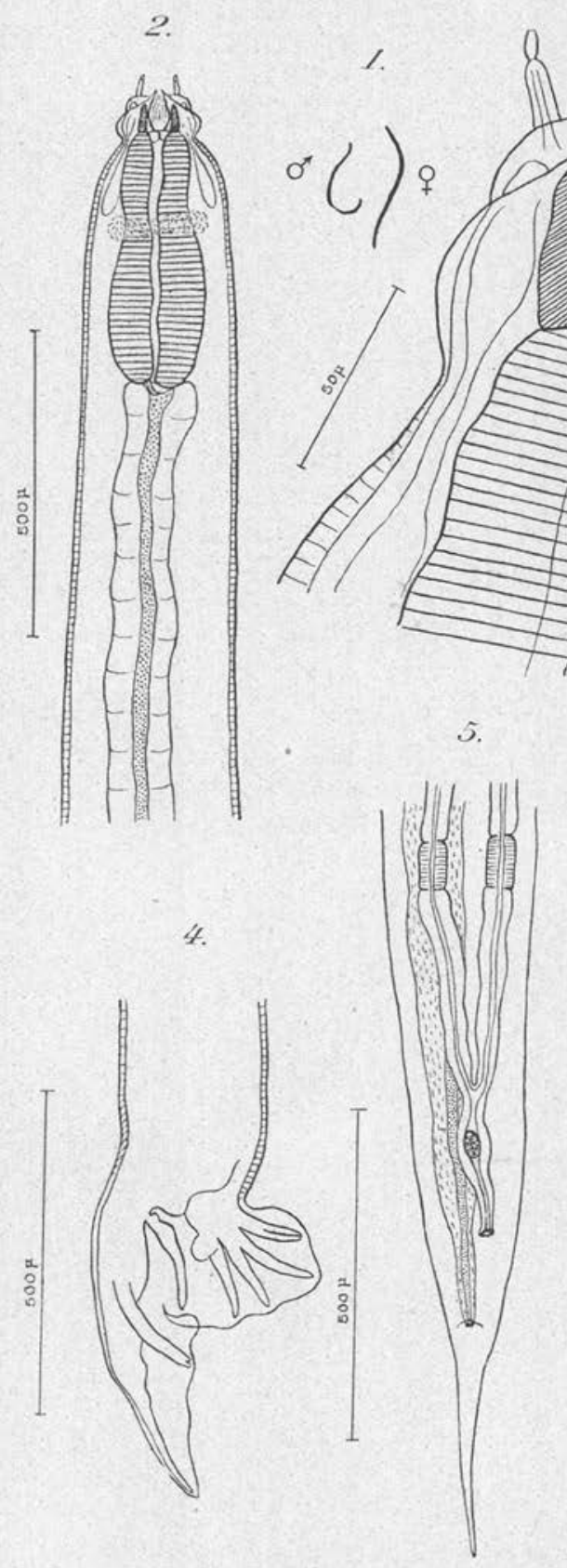

Tome II. PL. XIV.

Mémoire Neveu-Lejaike

3.
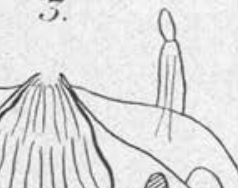

( -2
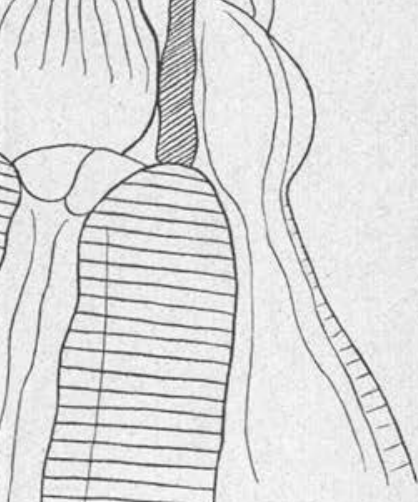

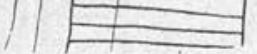




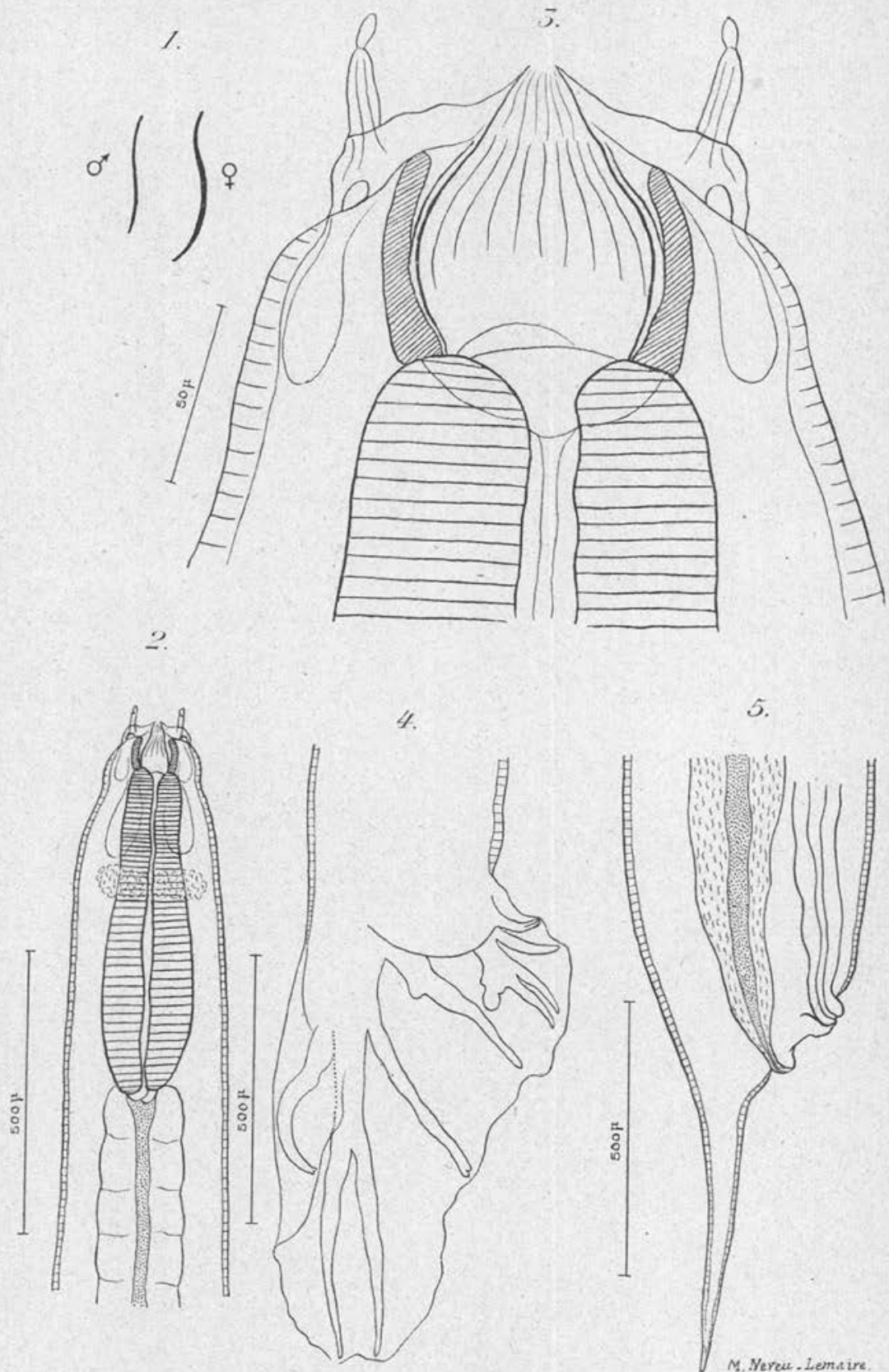

5.

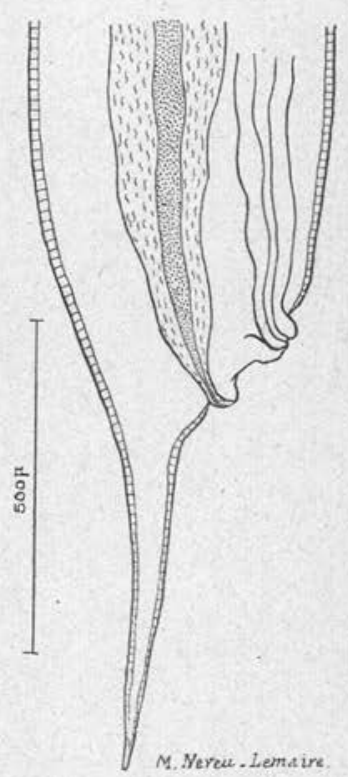

Henryella Bozasi n. g., n. sp. 

Tome II. PL. XVI.

MÉmotre Neveu-Lematre
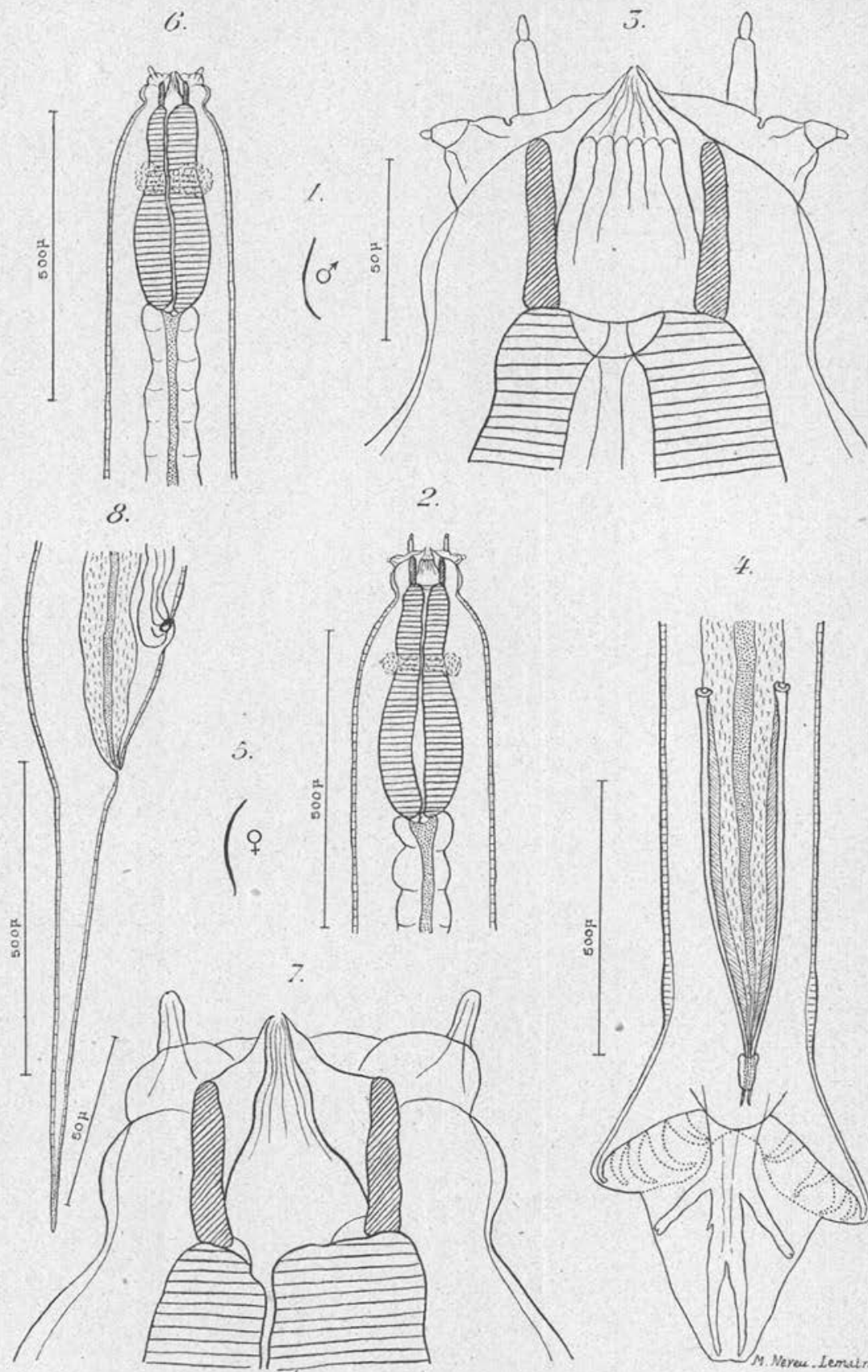

7.

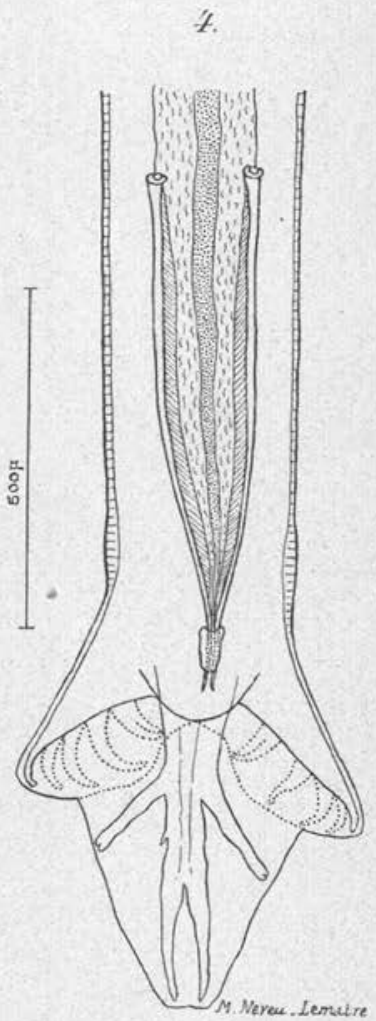

Henryella Zeltneri n. g., n. sp. (1 à 4)

Henryella Didieri n. g., n. sp. (5 à 7 ) 


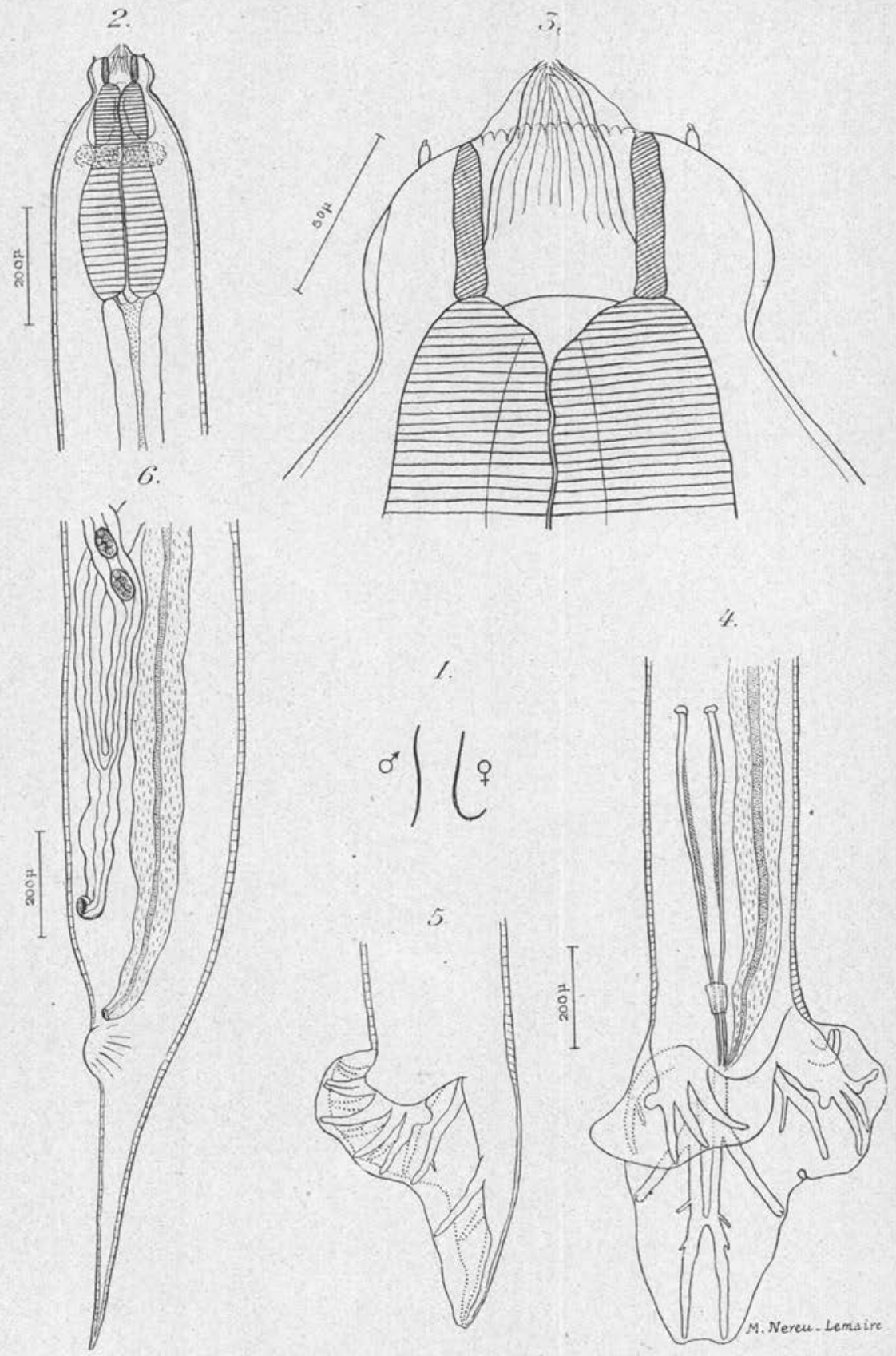

BUISSONIA RHINOCEROTIS n. g., n. sp. 

Mémoire Neveu-Lemaire
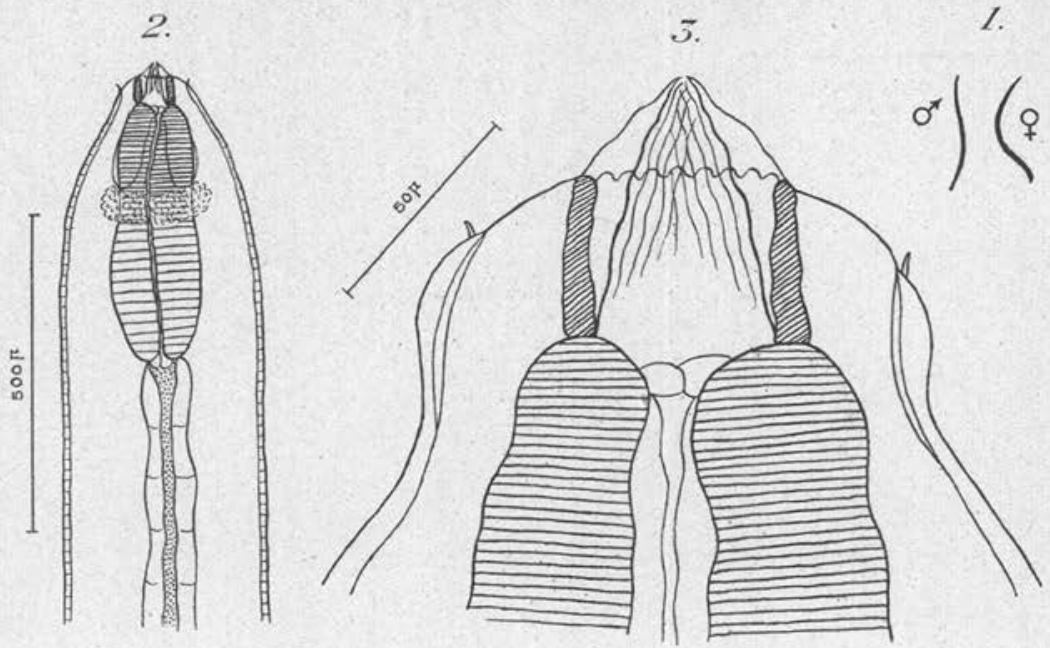

5.

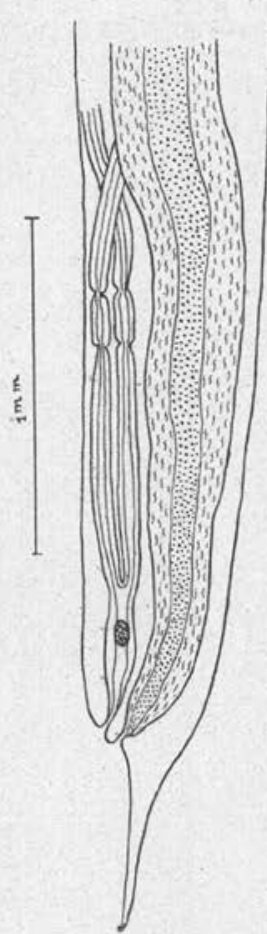

4.

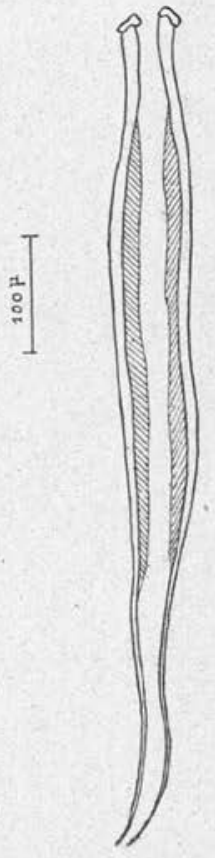

6.

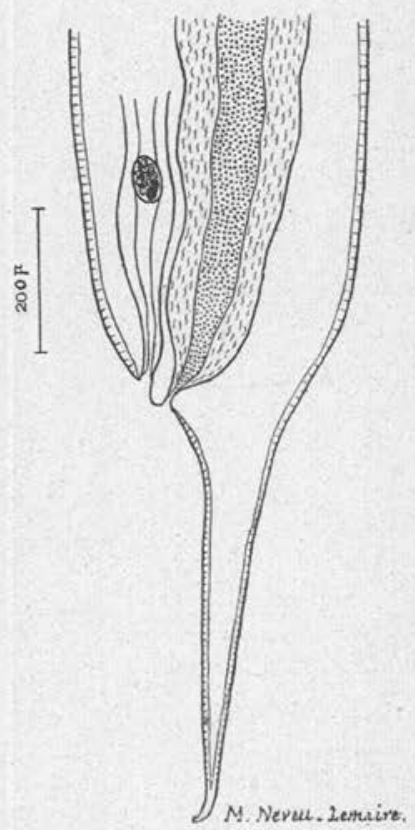

Buissonia AFricana n. g., n. sp. 


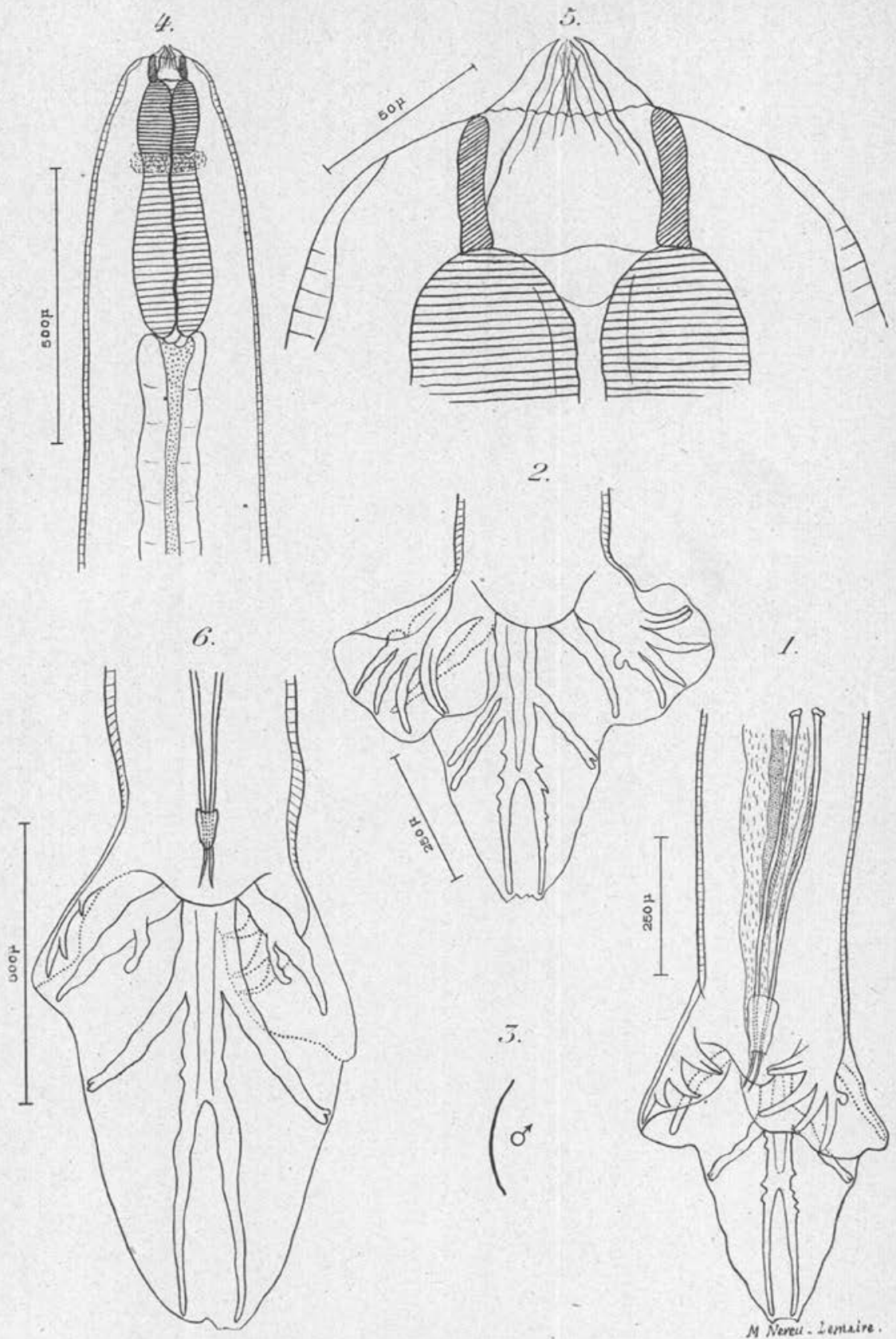

Buissonia africana n. g., n. sp. (1 et 2 ) Buissonia LONGIBURSA n. g., n. sp. (3 à 6 ) 

ANNALES DE PARASITOLOGIE

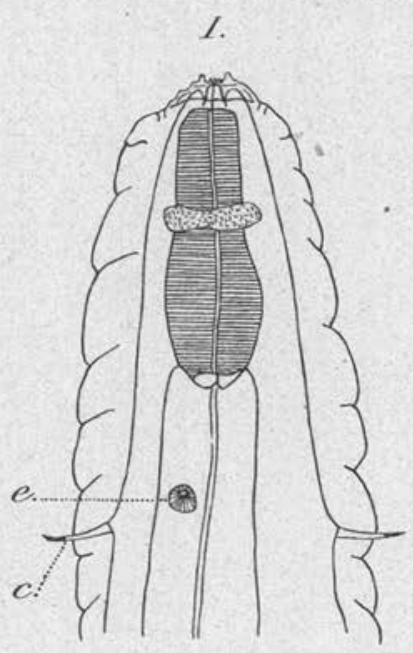

6.
Tome II. PL. XX.

MÉmoire Neved-Lemaire

7.

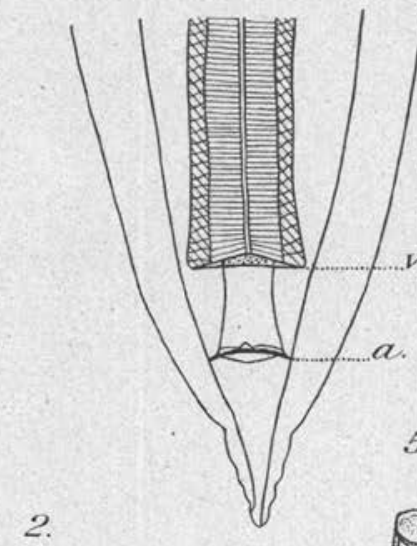

5.

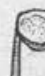

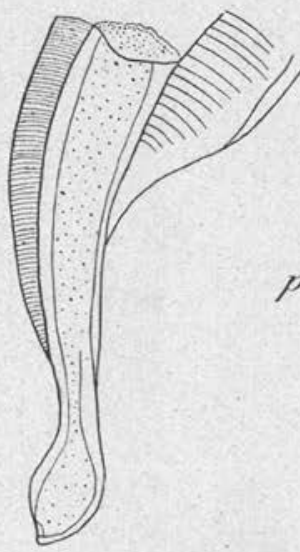

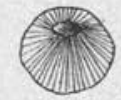

4.

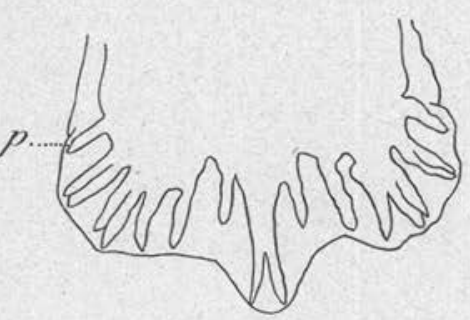

3.
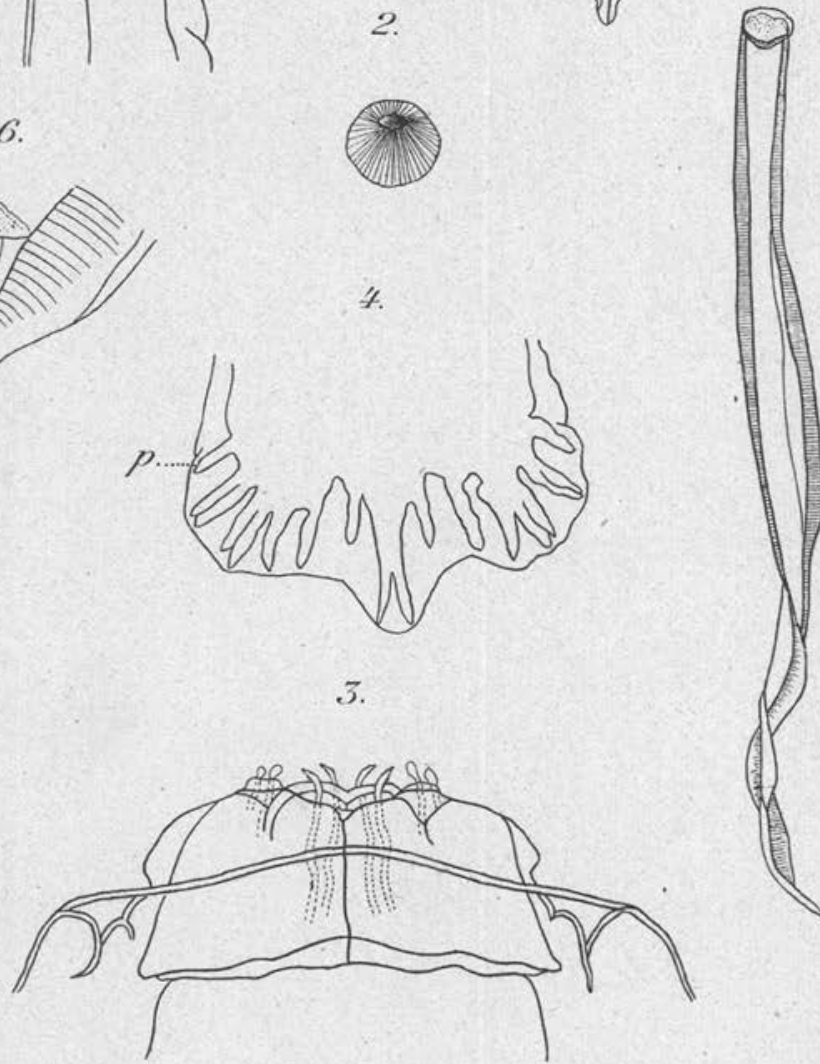

KILULUMa stYlosa (von Linstow, 1907), d'après Skrjabin 